\title{
A Systematic Review on Novel Mycobacterium tuberculosis Antigens and Their Discriminatory Potential for the Diagnosis of Latent and Active Tuberculosis
}

\author{
Noëmi R. Meier ${ }^{1,2}$, Marc Jacobsen ${ }^{3}$, Tom H. M. Ottenhoff ${ }^{4}$ and Nicole Ritz ${ }^{1,2,5 *}$ \\ ${ }^{1}$ University of Basel Children's Hospital, Mycobacterial Research, Basel, Switzerland, ${ }^{2}$ University of Basel, Faculty of \\ Medicine, Basel, Switzerland, ${ }^{3}$ Department of General Pediatrics, Neonatology, and Pediatric Cardiology, University \\ Children's Hospital, Heinrich Heine University, Düsseldorf, Germany, ${ }^{4}$ Department of Infectious Diseases, Leiden \\ University Medical Center, Leiden, Netherlands, ${ }^{5}$ The Royal Children's Hospital Melbourne, Infectious Disease Unit, \\ Melbourne, VIC, Australia
}

\section{OPEN ACCESS}

Edited by:

Christoph Hölscher, Forschungszentrum Borstel (LG)

Germany

Reviewed by:

Bernat Pérez de Val, Institut de Recerca i Tecnologia Agroalimentàries (IRTA), Spain

Sagarika Haldar,

Translational Health Science and Technology Institute, India

${ }^{*}$ Correspondence:

Nicole Ritz nicole.ritz@unibas.ch

Specialty section

This article was submitted to Microbial Immunology,

a section of the journal

Frontiers in Immunology

Received: 05 June 2018 Accepted: 08 October 2018 Published: 09 November 2018

Citation:

Meier NR, Jacobsen M, Ottenhoff THM and Ritz N (2018) A

Systematic Review on Novel Mycobacterium tuberculosis Antigens and Their Discriminatory Potential for the Diagnosis of Latent and Active Tuberculosis. Front. Immunol. 9:2476. doi: 10.3389/fimmu.2018.02476
Background: Current immunodiagnostic tests for tuberculosis (TB) are based on the detection of an immune response toward mycobacterial antigens injected into the skin or following an in-vitro simulation in interferon gamma-release assays. Both tests have limited sensitivity and are unable to differentiate between tuberculosis infection (LTBI) and active tuberculosis disease (aTB). To overcome this, the use of novel Mycobacterium tuberculosis (M. tuberculosis) stage-specific antigens for the diagnosis of LTBI and aTB has gained interest in recent years. This review summarizes current evidence on novel antigens used for the immunodiagnosis of tuberculosis and discrimination of LTBI and aTB. In addition, results on measured biomarkers after stimulation with novel M. tuberculosis antigens were also reviewed.

Methods: A systematic literature review was performed in Pubmed, EMBASE and web of science searching articles from 2000 up until December 2017. Only articles reporting studies in humans using novel antigens were included.

Results: Of 1,533 articles screened 34 were included in the final analysis. A wide range of novel antigens expressed during different stages and types of LTBI and aTB have been assessed. M. tuberculosis antigens Rv0081, Rv1733c, Rv1737c, Rv2029c, Rv2031 and Rv2628, all encoded by the dormancy of survival regulon, were among the most widely studied antigens and showed the most promising results. These antigens have been shown to have best potential for differentiating LTBI from aTB. In addition, several studies have shown that the inclusion of cytokines other than IFN- $\gamma$ can improve sensitivity.

Conclusion: There is limited evidence that the inclusion of novel antigens as well as the measurement of other biomarkers than IFN- $\gamma$ may improve sensitivity and may lead to a discrimination of LTBI from aTB.

Keywords: interferon gamma-release assay, immune-response, clinical studies, active tuberculosis, latent tuberculosis, cytokines 


\section{INTRODUCTION}

Tuberculosis (TB) was globally one of the top 10 causes of death in 2016 and more than 10 million new cases are estimated to occur each year (1). Accurate and early identification of latent tuberculosis infection (LTBI) has become one of the key strategies to reduce TB incidence in recent years $(1,2)$. This strategy is particularly important in those at risk for rapid progression from LTBI to active tuberculosis (aTB), which includes immunocompromised individuals and children (3).

The tuberculin skin test for many decades has been the standard immunodiagnostic test to detect LTBI, measuring the local response after intradermal injection of a purified protein derivative $(4,5)$. As the tuberculin skin test lacks specificity due to cross-reactivity with non-tuberculosis mycobacteria and the widely used vaccine strain Mycobacterium bovis Bacillus Calmette-Guérin (BCG), an in vitro antigen specific cytokinebased immuno-diagnostic test was developed in the 1990s $(6,7)$. The currently used interferon gamma-release assays include early secretory antigenic target (ESAT)- 6 and culture filtrate protein (CFP)-10 as stimulatory antigens. Both antigens are located in the region of difference 1 , which is absent in BCG and in most nontuberculosis mycobacteria rendering these tests more specific than the tuberculin skin test $(8,9)$. Interferon gamma-release assays have now in many setting replaced the tuberculin skin test for testing LTBI in adults and have been recommended for use in both resource-rich and resource-limited countries (10). Interferon gamma-release assays have two major limitations: limited sensitivity in children, particularly in those under 5 years of age, and inability to discriminate between LTBI and aTB (11-13).

Numerous studies have therefore aimed to identify other proteins from Mycobacterium tuberculosis (M. tuberculosis) different from ESAT- 6 and CFP-10 as potential candidates for inclusion in immunodiagnostic tests for TB. Several strategies employing in vitro, in vivo, and in silico approaches have led to the discovery of novel immunogenic proteins of M. tuberculosis (1416). An important milestone and the basis for these discoveries was the sequencing of the entire genome of $M$. tuberculosis in the late 90ies (17). To summarize the current state of research, we performed a systematic literature review on studies in humans that have included novel $M$. tuberculosis antigens for immunodiagnostic tests. The aim of this review was to compare current evidence on novel $M$. tuberculosis antigens for the diagnosis of LTBI and aTB and to identify antigens that are most promising to be included in further research. In addition, results on measured biomarkers after stimulation with novel M. tuberculosis antigens were also reviewed.

\footnotetext{
Abbreviations: CFP-10, $10 \mathrm{kDa}$ culture filtrate protein; DosR, dormancy of survival regulon; ELISA, enzyme-linked immunosorbent assay; ELISPOT, enzymelinked immuno-spot assay; EPTB, extrapulmonary tuberculosis; ESAT-6, 6 $\mathrm{kDa}$ early secretory antigenic target; FCM, flow cytometry; HBHA, Heparinbinding haemagglutinin; LTBI, latent tuberculosis infection; M. tuberculosis, mycobacterium tuberculosis; PBMC, peripheral blood mononuclear cell; QFT-GIT, quantiferon tuberculosis gold in tube; Rpf, resuscitation promoting factor; Rv, rough morphology virulent; $\mathrm{TB}$, tuberculosis.
}

\section{METHODS}

\section{Search Strategy}

A systematic literature search was performed guided by preferred reporting items for systematic reviews and meta-analyses (see PRISMA checklist Supplementary File 1). Studies measuring the immune response induced by novel $M$. tuberculosis antigens in patients with LTBI and aTB were included. PubMed, Embase and Web of Science (core collection) were searched for studies published between Jan 1st 2000 and Dec 31st 2017. Studies published before 2000 were excluded since interferon gammarelease assays have been marketed in the late 1990s. The PubMed search was done using medical subject heading terms. Search strings were generated for (i) tuberculosis, (ii) antigens, (iii) assay read-out and (iv) humans. Additional filters were included in the Embase and Web of science search (see Supplementary File 2 for details on search strategy). Articles in English, French or German were considered for inclusion. Additional studies were identified through references of publications identified for inclusion.

\section{Inclusion and Exclusion Criteria}

After initial screening (Figure 1) studies were evaluated for inclusion based on the following criteria (see also Supplementary File 3): (i) including patients with LTBI and/or aTB with details specified on classification of included patients comprising results from sputum microscopy, culture, polymerase chain reaction testing, radiography, and standard immunodiagnostic tests (interferon gamma-release assays or tuberculin skin test); (ii) further details of the study population available including age, gender, treatment, and testing for human immunodeficiency virus, (iii) immune response analyzed in blood samples for at least one novel antigen not currently or previously used in commercially available interferon gammarelease assays or tuberculin skin test (i.e. antigen other than ESAT-6, CFP-10, Tb7.7, purified protein derivative). If human immunodeficiency virus positive individuals were included, separate analysis of results from this patient group was required for inclusion. For pediatric studies, authors were contacted, if human immunodeficiency virus status was not specified in the original publication. Pediatric studies were included if children were human immunodeficiency virus negative or if human immunodeficiency virus was highly unlikely based on national prevalence of the study population. Studies including patients that have been treated with anti-tuberculous medication for 4 weeks or longer prior to testing were excluded from analysis. The search and selection of included studies was done by two authors (NM and NR). In unclear cases a shared decision for inclusion of the study was made.

\section{Data Extraction and Classification}

Data was extracted using a standardized form including the following variables: First author, year of publication; country; study population characteristics including age, patient group; details about methods used including cell type, incubation duration, type of antigen, assays used for read-out and main results. According to function, expression and location on 


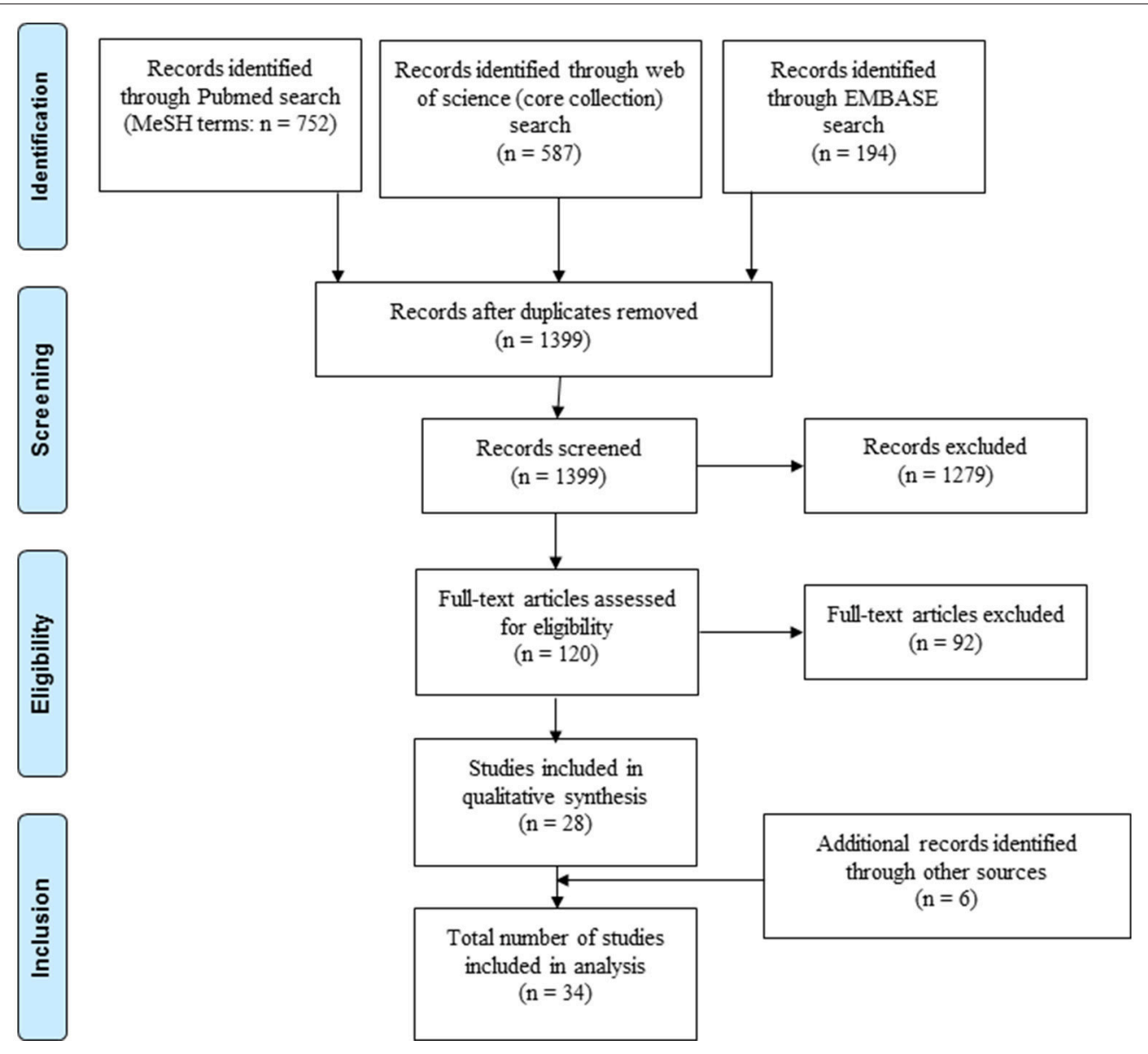

FIGURE 1 | Selection of articles included in the review.

the genome antigens were grouped into latency associated, reactivation- and resuscitation- associated or other.

\section{RESULTS}

\section{Included Studies}

A total of 1,533 records were identified through PubMed, Embase and Web of Science, of which 28 studies were included in the analysis (Figure 1). An additional six records were identified through cross-references of included articles resulting in a final number of 34 included studies. Reasons for exclusion of studies are summarized in Supplementary File 4.

\section{Characteristics of Included Studies}

Included studies originated from 16 countries with the majority $(12 / 34,35 \%)$ being from Europe (Table 1). A total of 5,084 patients were included of which 2,325 were classified as LTBI and 1,252 as aTB. In addition 1,507 healthy controls or other controls were included. The majority of studies were in adults only $(24 / 34,70 \%$; $)(18,20,21,23-27,29-32,35,36,38,39,42$, 45-51), 8/34 (24\%) studies were done in adults and children $(19,22,28,37,40,41,43,44)$ and $2 / 34(6 \%)$ were done in children only $(33,34)$. Outcomes were measured using enzymelinked immunosorbent assay (ELISA), enzyme-linked immunospot assay (ELISPOT) and flow cytometry (FCM) in 27, 6 and 12 studies, respectively (Table 1), and some studies used several different assays for outcome measurement. IFN- $\gamma$ was measured as the only outcome in $16 / 34$ (47\%) studies. $18 / 34$ (53\%) studies included further cytokines in the analysis most commonly being IL-2, IL-10, IL-17, and TNF- $\alpha$ (Table 2).

\section{Patient Groups Investigated}

Patient groups in the studies included aTB, LTBI, exposed, healthy, and sick control patients (Tables 1, 3). Criteria for patients with aTB were similar and mostly consistent across studies including culture confirmation and/or presence of acid fast bacilli in sputum smear. Two studies also used positive polymerase chain reaction results as confirmation of $\operatorname{aTB}(34,37)$. Further to this in $6 / 34$ (18\%) studies diagnosis of aTB was based on clinical criteria $(20,34,37,40,50,51)$. Definitions for LTBI patients were heterogeneous and highly variable across studies. Overall 14/34 (41\%) studies included a control group of healthy participants who had negative test results for interferon gammarelease assays /tuberculin skin test and/or no known history of 


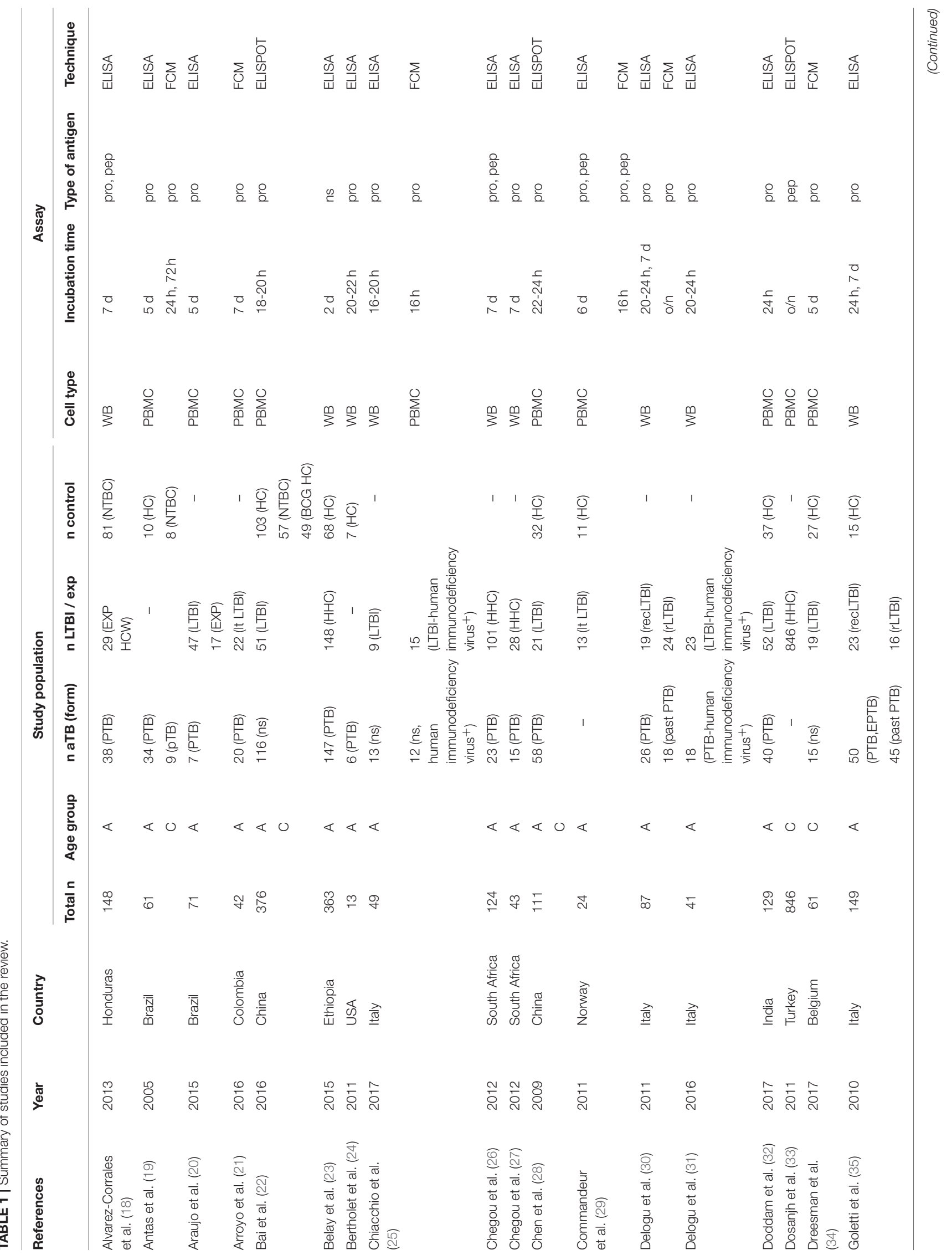




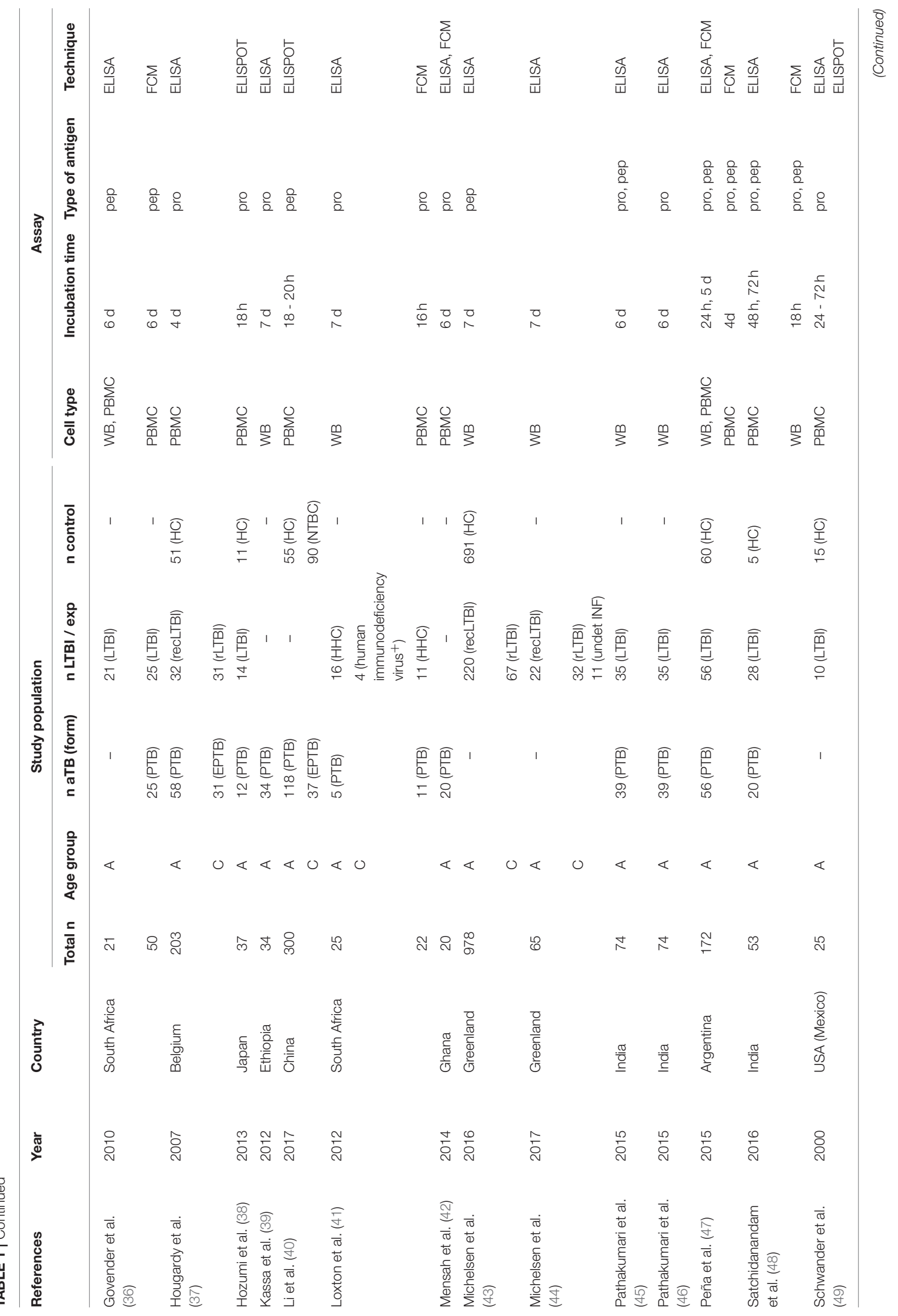




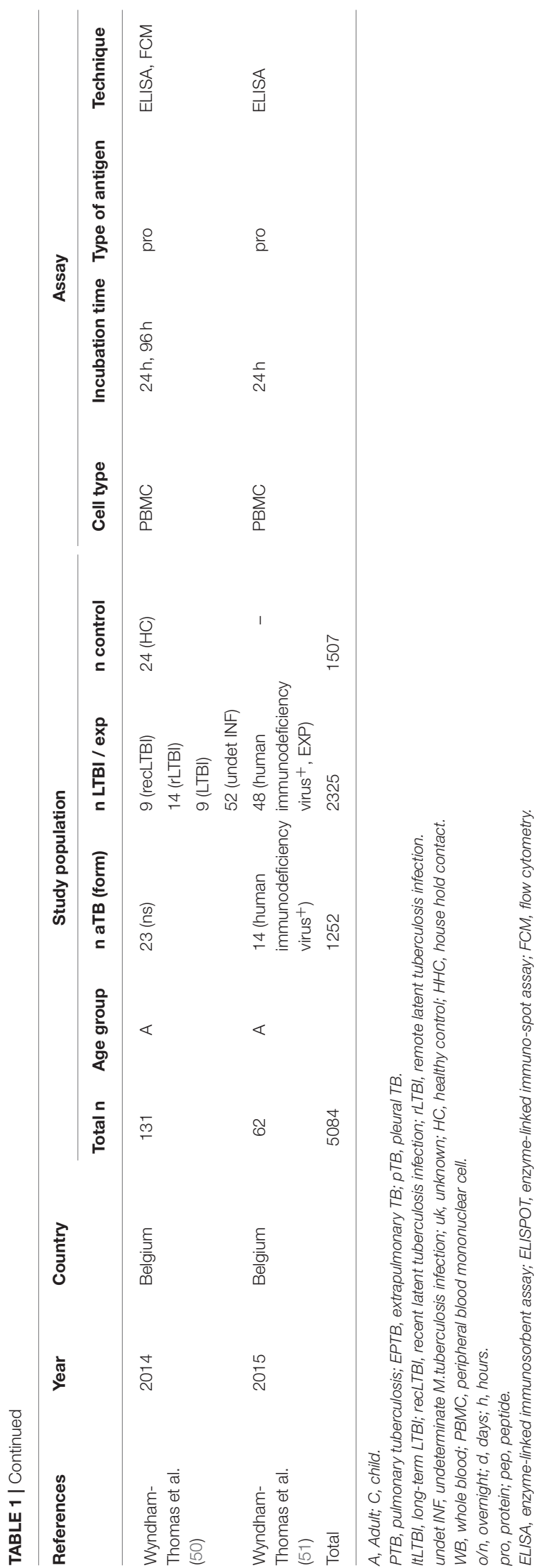

or exposure to aTB $(19,22,24,28,29,32,34,35,37,38,43,47$, $48,50)$. A further $2 / 34$ (6\%) studies included exposed individuals into the control group as long as they stayed healthy (e.g., no symptoms and microbiologically negative or tuberculin skin test negative) $(32,34)$. Another $2 / 34(6 \%)$ studies included a healthy control group but either no interferon gamma-release assays /tuberculin skin test was done or the community controls were tuberculin skin test positive (no exposure) $(40,49)$. Finally, $1 / 34$ (3\%) study included patients with a respiratory disease other than TB as a control group (18) and 3/34 (9\%) included both a healthy and a sick control group $(19,22,40)$. A single patient group was evaluated by $4 / 34$ (12\%) studies being either: active pulmonary TB $(39,42)$, pediatric household contacts from TB index cases (33), or LTBI patients (44).

\section{Types of Antigens Investigated}

Over 300 individual novel M. tuberculosis antigens were tested among the studies included in this review. Table 4 shows a selection of the most commonly reported 92 antigens that were reported in these studies. Of those Rv2031c, Rv2029c, antigens of the Ag85 complex, Rv0475 (HBHA), Rv2628, Rv1733c, Rv1737c, Rv0081, Rv2032, Rv0867c, and Rv2389c are among the most frequently tested ones. Most antigens tested (48 antigens; Rv2031c, Rv2029c, Rv2628, Rv1733c, Rv1737c, Rv0081, Rv2032) belong to the group of latency associated antigens. The majority of these $42 / 48(88 \%)$ are part of the dormancy of survival regulon (DosR), a region in the genome comprising approximately 50 genes expressed during latency. Fewer antigens (e.g., Rv0867c, Rv2389c) belong to the group of resuscitation associated antigens, which contains 5 different genes encoding resuscitation promoting factors (Rpfs). Table 5 summarizes the most signicant findings for the most important antigens assessed in the studies grouped according to family of antigens.

\section{Latency Associated Antigens Dormancy of Survival Regulon}

Two studies by Chegou et al. and Kassa et al. assessed the immune response against a wide range of stage-specific antigens, including serval antigens of the DosR regulon $(26,39)$. Both studies used stimulated whole blood to assess IFN- $\gamma$ response in a long-term assay. Kassa et al. identified Rv0081, Rv1733c and Rv2006 among the most immunogenic antigens stimulating high concentrations of TNF- $\alpha$, IL-10, and IL-6 in aTB (39). Chegou et al. reported significant differences in IFN- $\gamma$ responses for a number of DosR antigens including Rv1735c, Rv2006, Rv2625c, Rv1996, Rv2032, Rv2629, Rv3126c, Rv0081, Rv2631, Rv3130c, Rv2624c, Rv2007c, Rv2028c, and Rv3134 between exposed and aTB (26). Based on these results a selection of these antigens was tested in a further study which also measured additional cytokines. Stimulation with Rv0081, Rv2032, and Rv1737c had the highest discriminatory potential of aTB vs. exposed when IL-12(p40), IP10 , IL-10 and TNF- $\alpha$ were analyzed (27). A study by Mensah et al. evaluated the immune response after stimulation with Rv1733c, Rv2029c, and Rv2628 to monitor treatment response in aTB patients (42). Concentrations of several biomarkers including 
TABLE 2 | Summary of cytokines measured in studies included in this review (all cytokines listed).

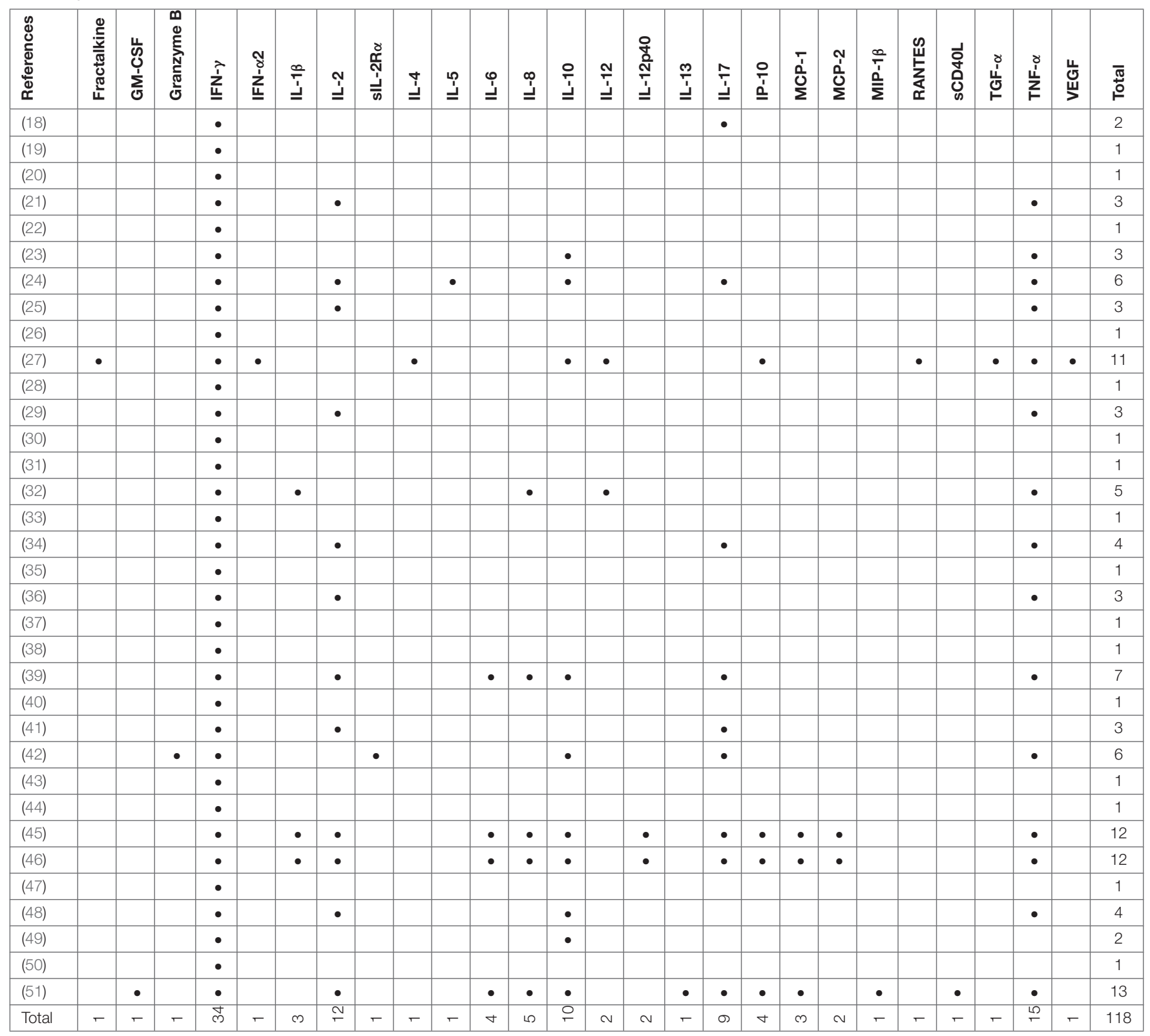

IFN- $\gamma$, Granzyme B, IL-17, and sIL-2R $\alpha$ increased during antimycobacterial treatment but only Rv1733c-specific Granzyme B levels were significantly increased compared to baseline levels. Hozumi et al. found 6 out of 33 tested DosR antigens (Rv0570, Rv1996, Rv2004c, Rv2028c, Rv2029c, and Rv3133c) relevant for differentiation of aTB and LTBI as these induced higher concentrations of IFN- $\gamma$ in LTBI (38).

Other DosR antigens such as Rv2031c showed conflicting results. For example, Belay et al. found that short-term stimulation with Rv2031c resulted in significantly lower IFN- $\gamma$, TNF- $\alpha$, and IL-10 concentrations in aTB patients compared to TB exposed individuals and healthy controls at baseline and over a 12-month follow-up period (23). Conversely, Goletti et al. and Hozumi et al. did not find differences in IFN- $\gamma$ concentrations upon Rv2031c stimulation in aTB, LTBI patients and controls $(35,38)$. Loxton et al. looked at IFN- $\gamma$ concentrations in 7 days Rv2031c-stimulated whole blood and found no differences between aTB and household contacts (41).

Commandeur et al. showed stimulation with Rv1733c, Rv2029c and Rv2031c resulted in an increase of double and single cytokine-producing T cells among LTBI patients compared to healthy controls. Particularly IFN- $\gamma /$ TNF- $\alpha$-producing CD8 T cells were the most frequently found subset (29). This supports findings from a study by Arroyo et al. that reported stimulation with Rv1737c and Rv2029c increased IFN- $\gamma$ - and/or TNF- $\alpha$ producing CD4 and CD8 T cells in patients with LTBI compared to aTB (21). IFN- $\gamma$ production in response to Rv2029c was also significantly increased in LTBI compared to aTB patients and 
Meier et al.

Novel MTB Antigens in TB Diagnostics

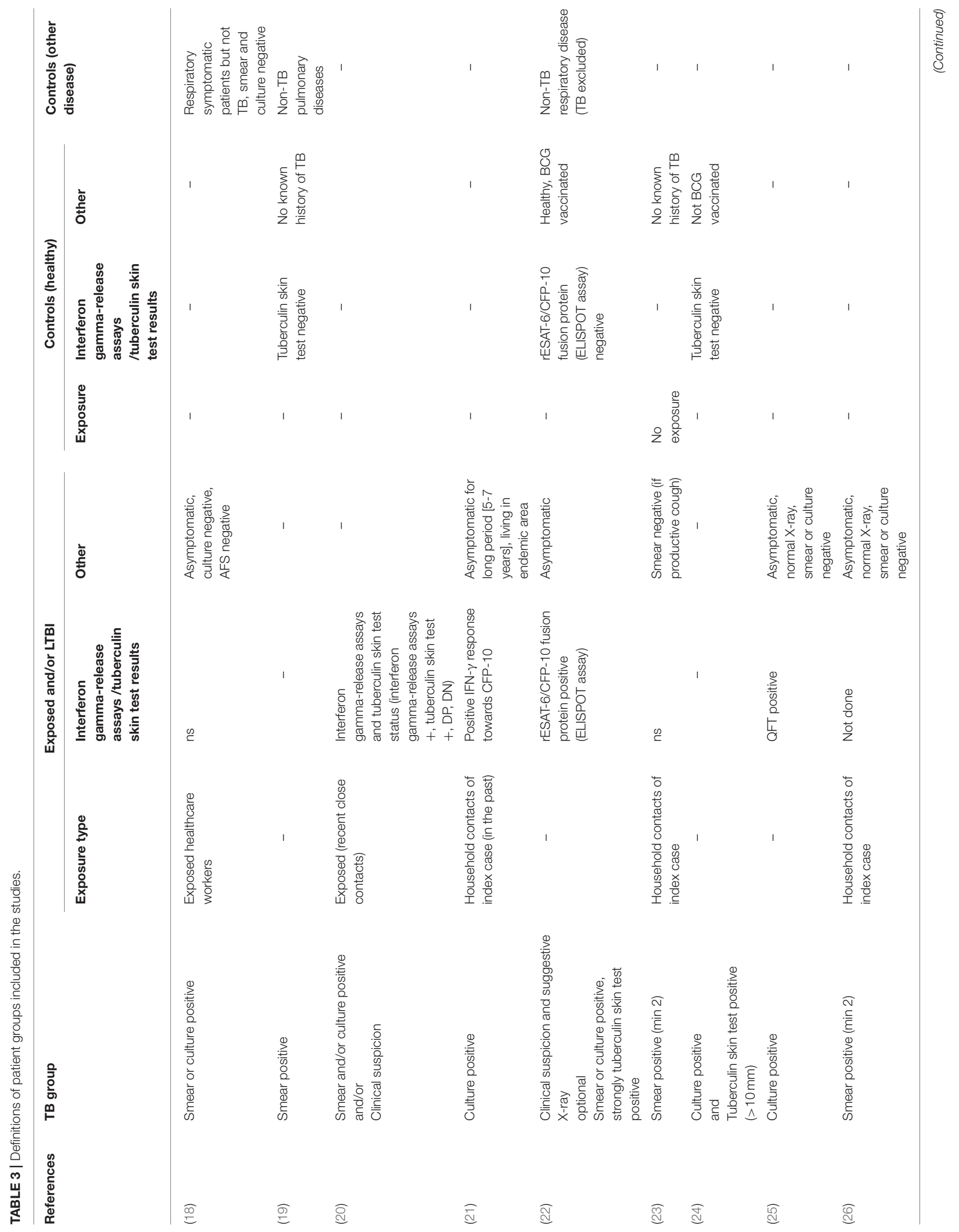

Frontiers in Immunology | www.frontiersin.org

8

November 2018 | Volume 9 | Article 2476 


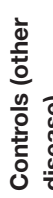

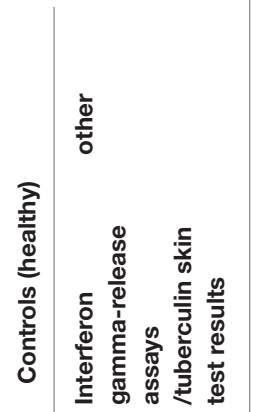

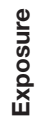

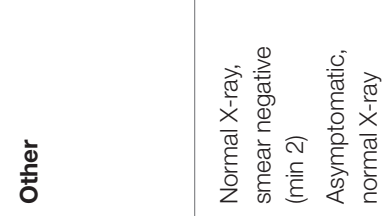

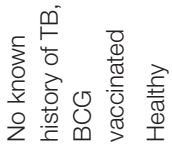

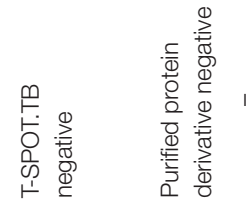

일

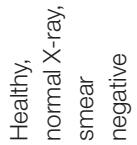

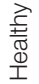

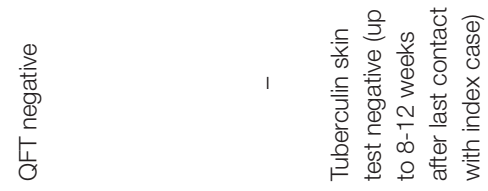

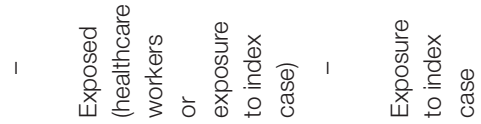

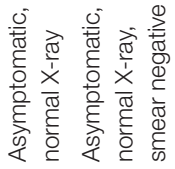

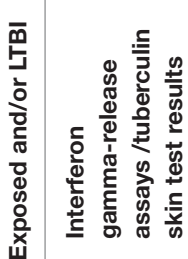

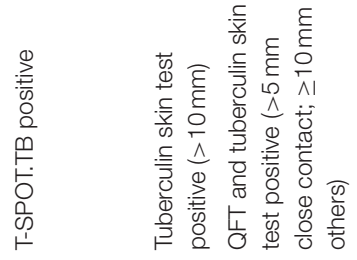

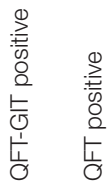

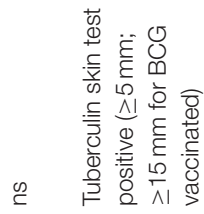

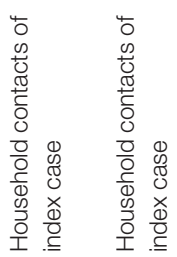
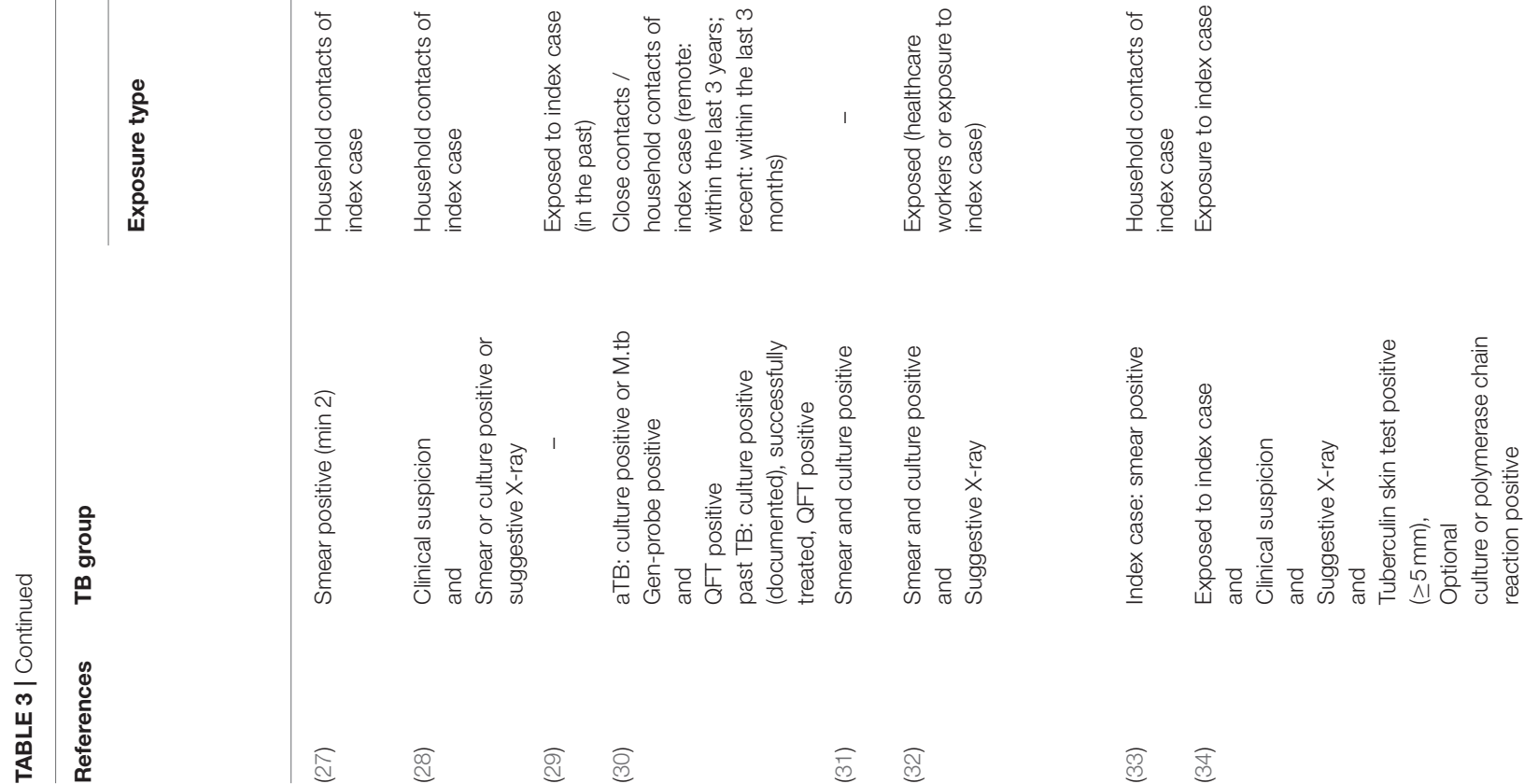


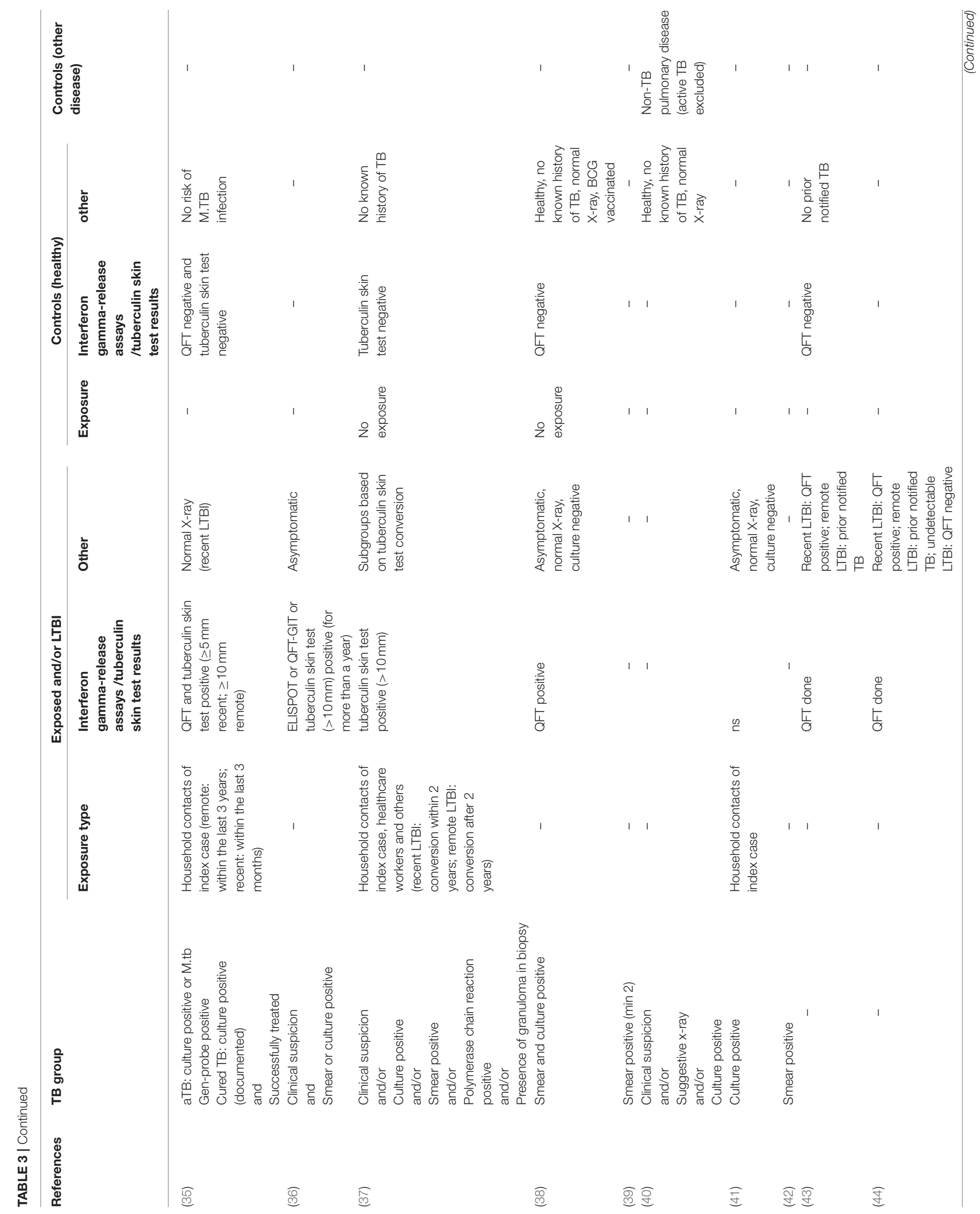




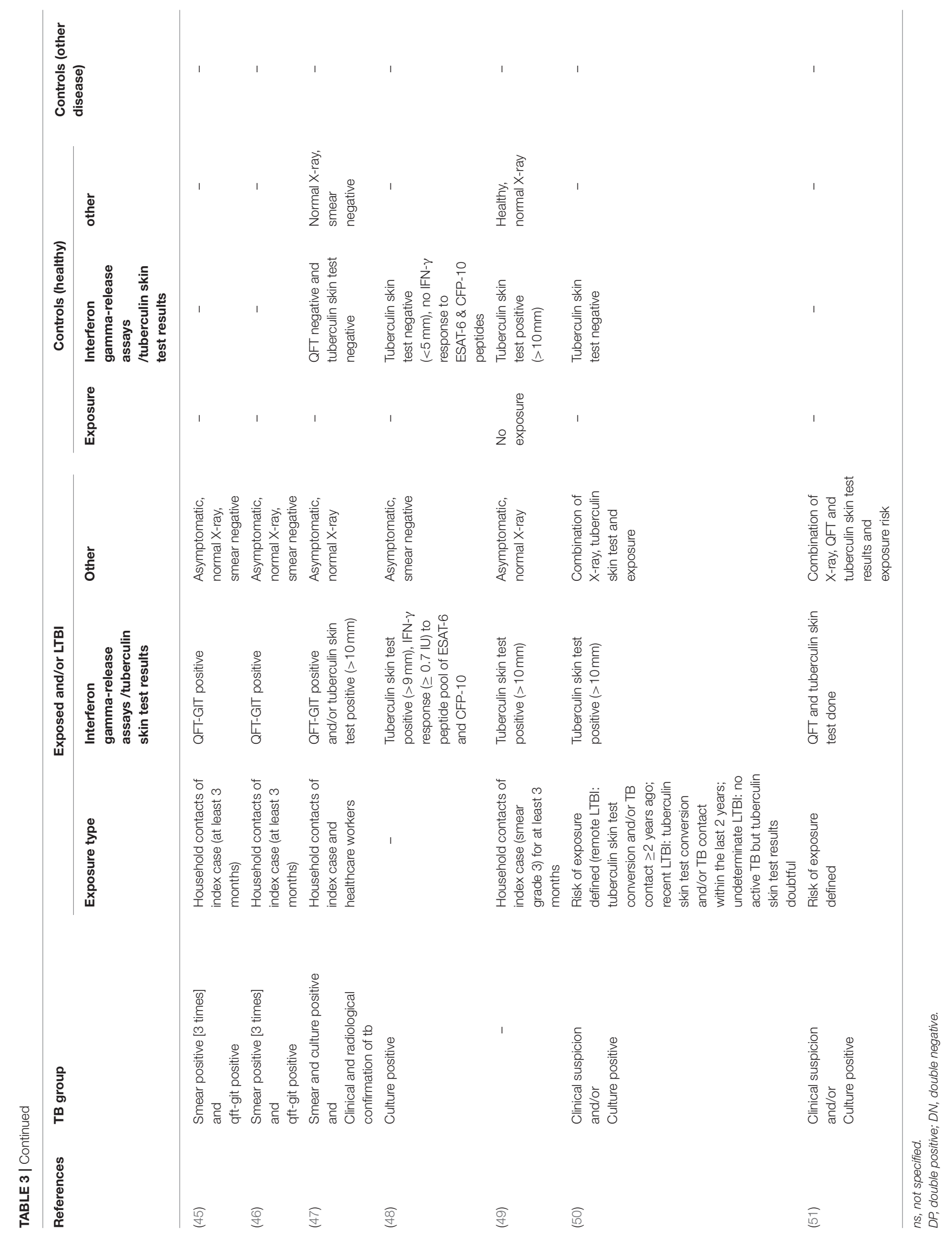


Meier et al.

Novel MTB Antigens in TB Diagnostics

TABLE 4 | Summary of antigens used in the studies included in this review.

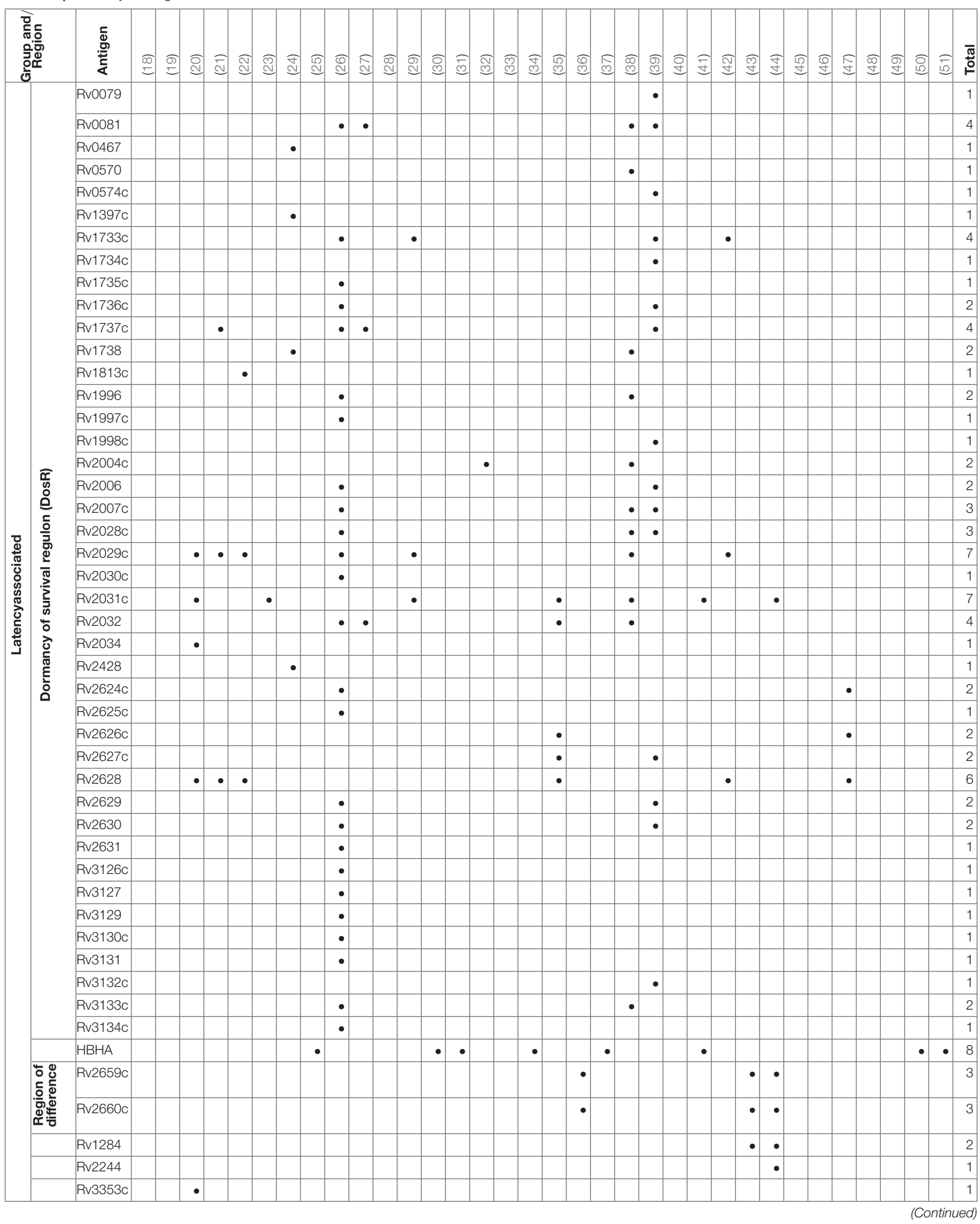

Frontiers in Immunology | www.frontiersin.org

12

November 2018 | Volume 9 | Article 2476 


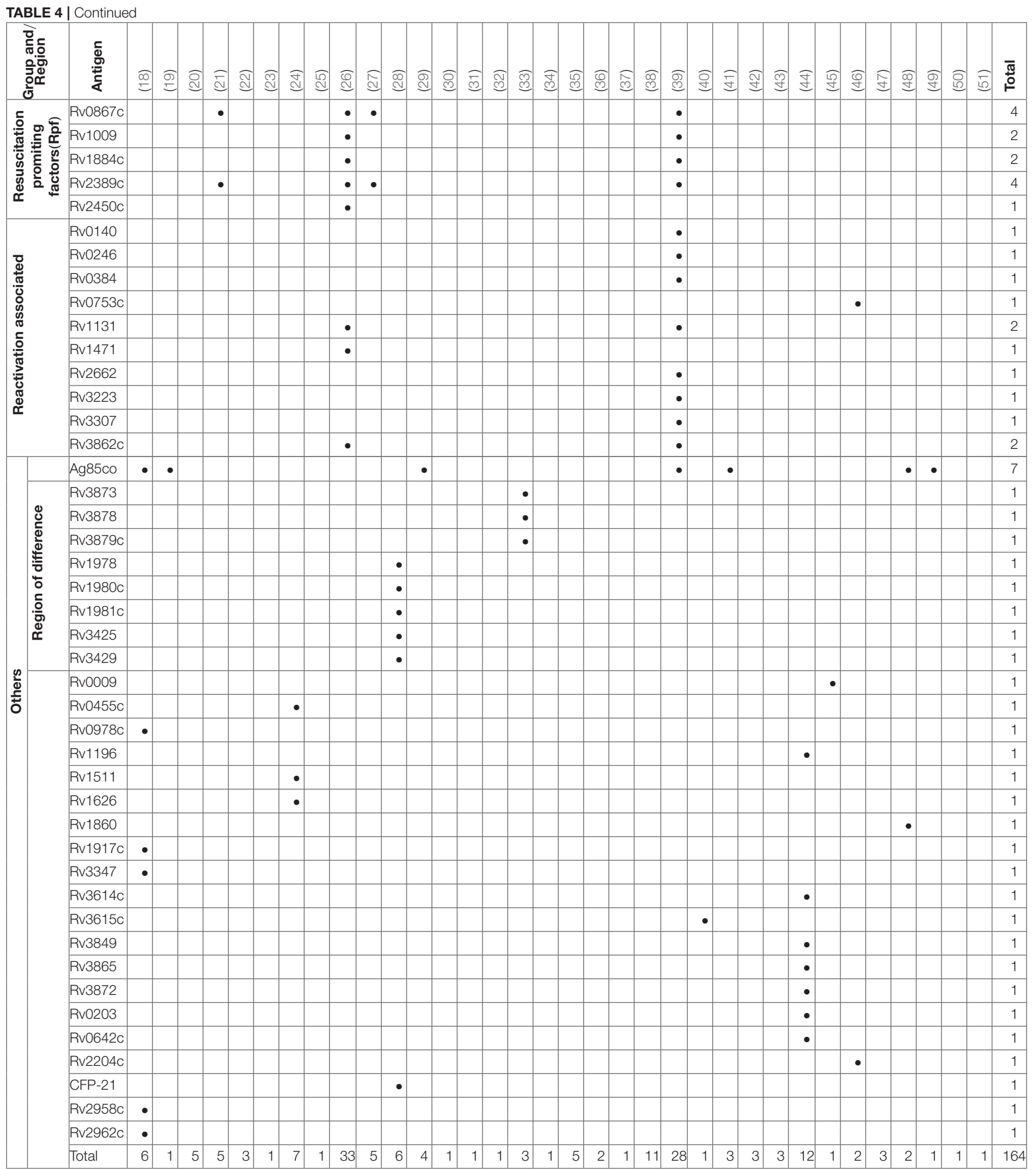

healthy controls in a study by Bai et al. (22). This study also included a control group with respiratory disease and showed that the response to latency antigens Rv2029c, Rv2628, Rv1813c was negligible in these patients. Araujo et al. used Rv2029c, Rv2031c and Rv2034 and found an increased IFN- $\gamma$ response upon stimulation in the LTBI group; the three antigens combined were able to detect $95 \%$ of LTBI patients (20).

Doddam et al. characterized the immune-response toward Rv2004c and found that this antigen elicits a strong proinflammatory (TNF- $\alpha$, IL-8, IL-1 $\beta$, and IL-12) response in LTBI 


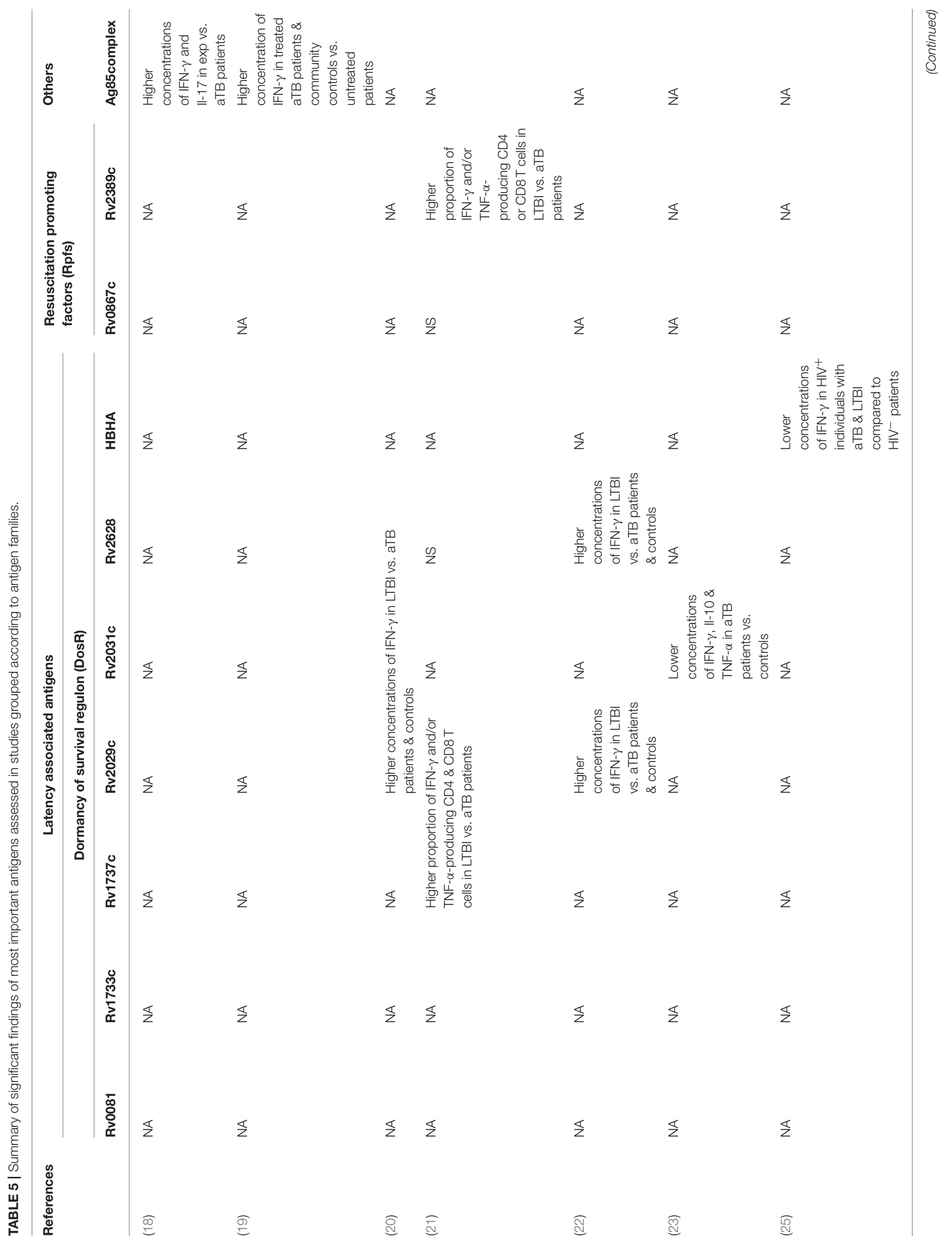




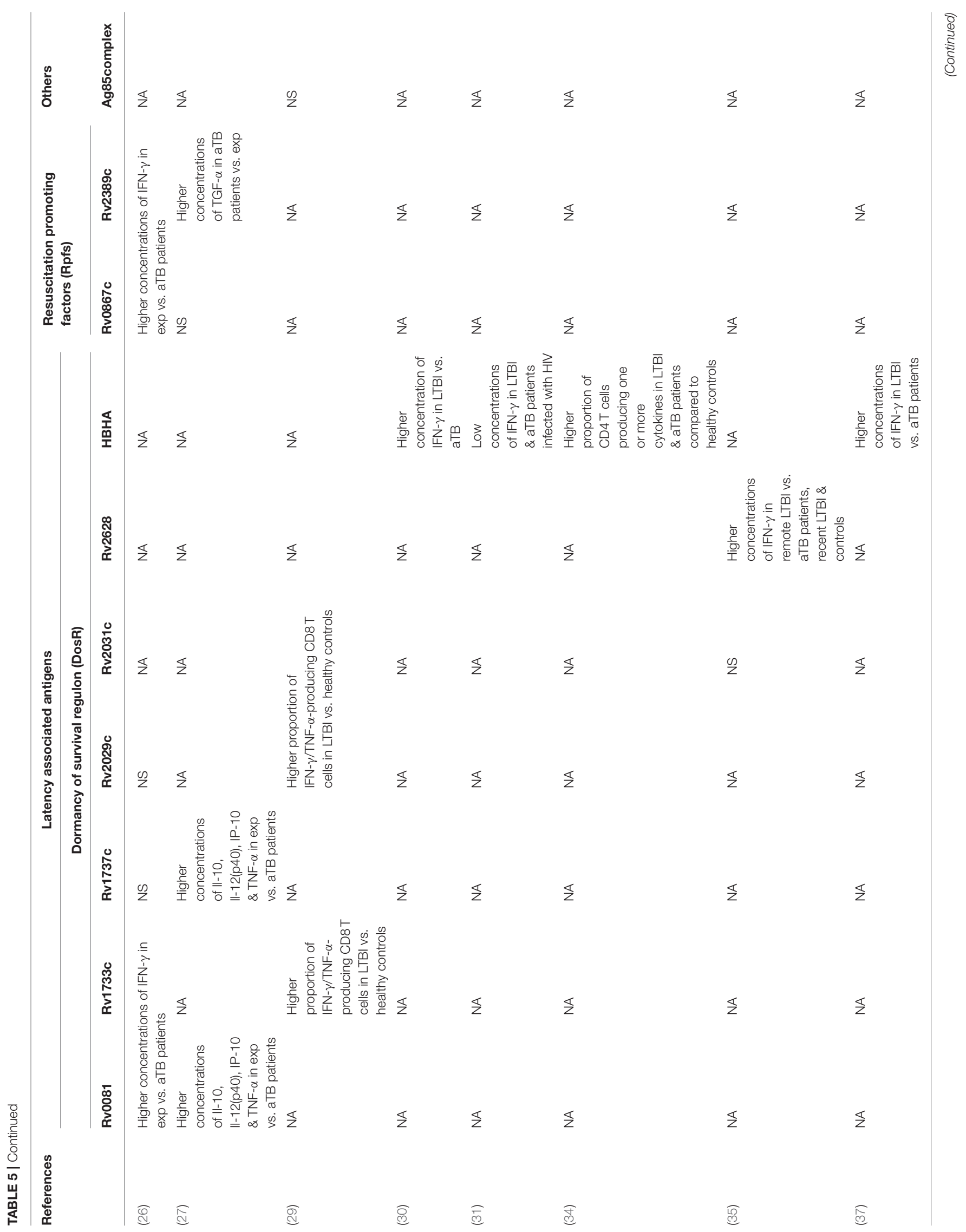


Meier et al.

Novel MTB Antigens in TB Diagnostics

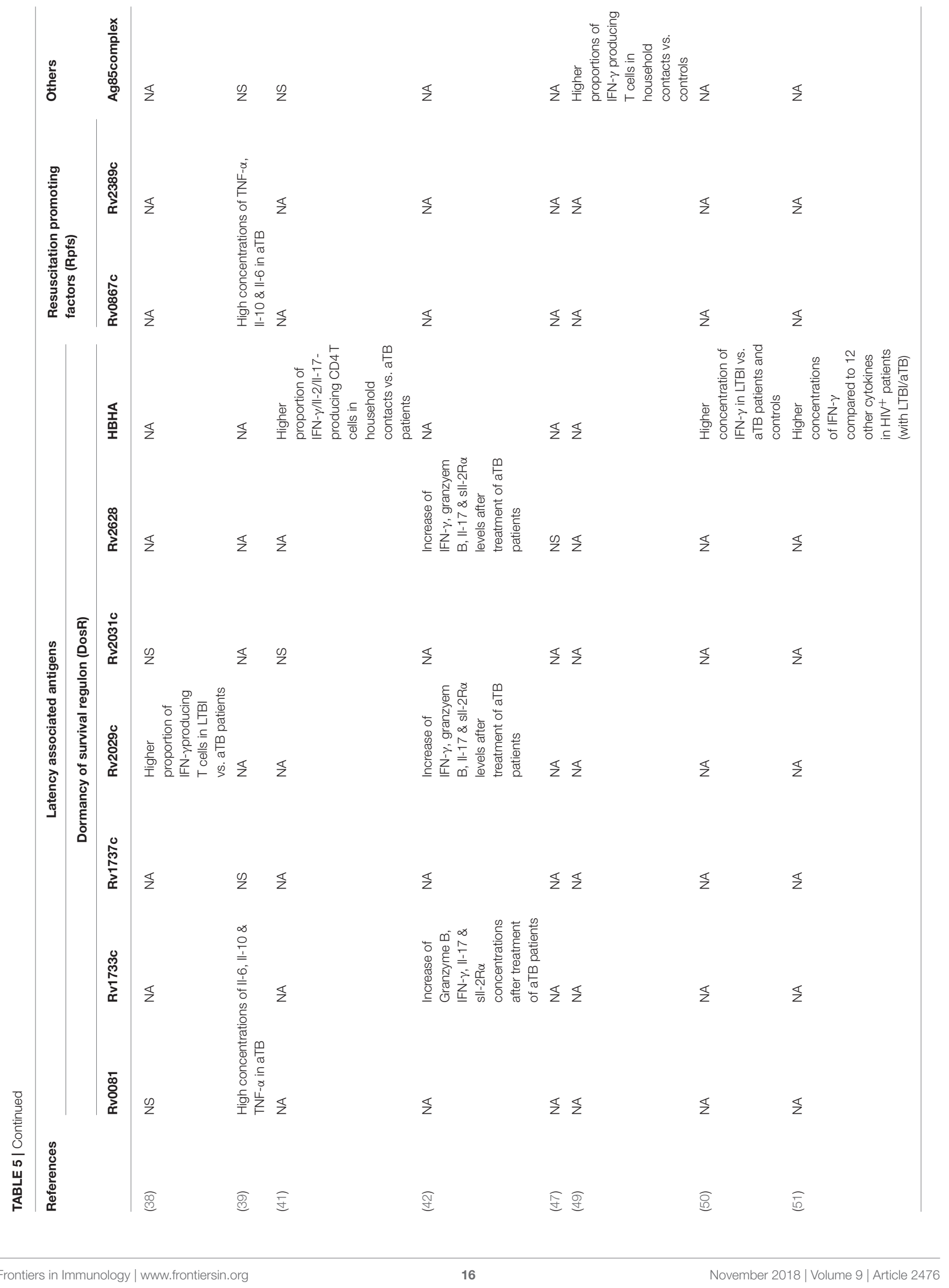


patients compared to aTB and healthy controls (32). Similarly, Hozumi et al. showed that Rv2004c elicits increased IFN- $\gamma$ producing $\mathrm{T}$ cells in a population of LTBI patients compared to aTB and controls in Japan (38).

Response to latency antigens Rv2624-30 were assessed in several studies. Goletti et al. found stimulation with Rv2628 to result in high concentrations of IFN- $\gamma$ which the authors associated with protection against aTB in those patients. Rv2626c and Rv2627c, however, showed no significant differences in LTBI vs. aTB patients (35). Araujo et al. and Bai et al. also measured higher IFN- $\gamma$ responses in peripheral blood mononuclear cells stimulated with Rv2628 in LTBI compared to aTB patients and healthy controls $(20,22)$. In contrast, results from Peña et al. suggest Rv2626c, but not Rv2624c and Rv2628 to be a strong inducers of IFN- $\gamma$ in LTBI compared to aTB patients and healthy controls (47). Chegou et al. demonstrated that Rv2624c as well as Rv2625c induced significantly higher IFN- $\gamma$ responses in exposed household contacts compared to aTB patients (26). In a study by Kassa et al, in aTB patients Rv2627c, Rv2629, and Rv2630 were among the most immunogenic antigens inducing high concentrations of several cytokines (including TNF- $\alpha$, IL-6, IL10) (39). Bertholet et al. assessed the effect of anti-mycobacterial treatment in aTB patients and found generally low levels of IFN$\gamma$ in response after treatment initiation. However, Rv2624 was among the few antigens that showed an increase in stimulated IFN- $\gamma$ concentrations over the course of the treatment (24).

\section{Heparin-Binding Hemagglutinin (HBHA, Rv0475)}

A study by Loxton et al. measuring intracellular cytokines after stimulation with HBHA found IFN- $\gamma /$ IL-2/IL-17-producing CD4 T cells to be increased in household contacts compared to aTB. Interestingly, in the same study IFN- $\gamma$ concentrations measured in the supernatant of a 7-day stimulation assay using HBHA did not differ among both groups (41). Similarly, Delogu et al. tested recombinant methylated HBHA in patients with LTBI and aTB and found an increased IFN $-\gamma$ response in patients with LTBI in both short-term and long-term stimulation assays (30). Likewise Hougardy et al. were able to show improved sensitivity compared to ESAT-6 and purified protein derivative for identification of LTBI in a low-endemic setting using a 4-day stimulation with HBHA (37). Wyndham-Thomas et al. explored the potential of a $24 \mathrm{~h}$ and $96 \mathrm{~h}$ stimulation with HBHA for LTBI diagnosis in a low-TB-endemic setting and confirmed the diagnostic potential of the shorter incubation in detecting recent and remote LTBI compared to a commercial interferon gamma-release assays (50). In another study by the same authors including human immunodeficiency virusinfected patients they found a $24 \mathrm{~h}$ stimulation with HBHA induced higher concentrations of IFN- $\gamma$ compared to 12 other cytokines measured (51). A further study including human immunodeficiency virus-infected patients by Delogu et al. showed that the absence of response toward HBHA correlated with increased risk of developing aTB indicating that a response to HBHA may be a correlate for protection (31). Chiacchio et al. characterized the $\mathrm{T}$ cell response toward HBHA among human immunodeficiency virus-infected and -uninfected patients with LTBI and aTB and showed IFN- $\gamma$ production by CD4 T cells to be generally lower in the human immunodeficiency virusinfected individuals (25). Dreesman et al. reported stimulation with HBHA in children and found that antigen induced CD4 T cells producing at least one cytokine to be significantly higher in both LTBI and aTB. Healthy children in that study showed a negligible cytokine response. In addition they showed that HBHA induced IL-17-procuding CD4 T cells only in young children below 3 years of age with LTBI but not in aTB patients and older children, suggesting that age may also influence the performance of certain antigens (34).

\section{The Starvation Regulon}

The starvation regulon are a set of genes upregulated by M. tuberculosis in response to nutrient deprivation (52). Two examples that have been tested in a number of studies included in this review are Rv2659 and Rv2660. In a study by Govender et al. long-term stimulation of PBMCs with Rv2659 and Rv2660 resulted in an increase of cytokine production and proportions of IFN- $\gamma /$ TNF- $\alpha /$ IL-2-producing polyfunctional CD4 T cells in LTBI compared to aTB. However, differences were more distinct upon stimulation with ESAT-6/CFP-10 and BCG. No differences were seen for the CD8 T cell population as proliferation and cytokine expression was generally low (36). Two studies in Greenland looking at remote and recent LTBI found that stimulation with Rv2659 and Rv2660 resulted in variable IFN- $\gamma$ responses over the course of infection and were not associated with protection against disease progression. Furthermore, positive responses for those two antigens were also frequently observed in patients that tested negative in commercial interferon gamma-release assays, which questions the reliability of those findings $(43,44)$.

\section{M. tuberculosis Reactivation-Associated Antigens and Resuscitation Promoting Factors}

Kassa et al. and Chegou et al. assessed immune responses to five Rpfs including Rv0867c, Rv1009, Rv1884c, Rv2389c, Rv2450c $(26,39)$. Stimulation with all five Rpfs induced significantly higher IFN- $\gamma$ responses in household controls compared to aTB index cases. The two "reactivation-associated antigens" Rv1131 and Rv1471 were frequently recognized in household controls and showed significant differences resulting in increased INF$\gamma$ production in household contacts compared to aTB (26). Additional markers and cytokines were explored in a followup study and showed the diagnostic potential of Rv2389cspecific TGF- $\alpha$ concentrations being the most promising in discriminating aTB (27). Kassa et al. showed four Rpfs to be the most immunogenic and found high concentrations of additional cytokines (e.g., IL-6, IL-10, and TNF- $\alpha$ ) in aTB patients (39). A study in Colombia showed that T cell responses toward Rv2389c were both mono- or bifunctional with increased frequencies of IFN- $\gamma$ and/or TNF- $\alpha$-producing CD4 and CD8 T cells in LTBI compared to aTB patients (21). Pathakumari et al. were able to demonstrate the discriminatory potential of Rv0753c specific 
IFN- $\gamma$ and IFN- $\gamma$ /TNF- $\alpha$ responses to identify LTBI and aTB patients (46).

\section{Other M. tuberculosis Antigens Region of Difference Encoded Antigens}

Dosanjh et al. investigated the role of several region of difference 1 encoded antigens in an ELISPOT assay in a study among children that were household contacts. Rv3873, Rv3878, and Rv3879c were included as additional antigens to improve sensitivity of the standard ELISPOT assay. Rv3873 and Rv3878 were found to be predictors of disease progression from LTBI to aTB (33).

Chen at al. tested a number of region of difference 2- and region of difference 11-encoded antigens for their potential of diagnosing LTBI in a BCG-vaccinated population (28). The immune response toward Rv1978, Rv1980c, Rv1981c, Rv3425, Rv3429, and Rv1984c was tested in an ELISPOT assay in LTBI and aTB patients as well as healthy controls. Rv1980c induced the highest frequency of IFN- $\gamma$ producing T cells. However, none of the novel antigens tested did improve sensitivity compared to the commercial ELISPOT assay. Combining the read-out including Rv3425, Rv1981c and the region of difference1-encoded antigens (ESAT-6, CFP-10) however increased sensitivity for detecting aTB.

\section{Antigens of the Ag85 Complex (Ag85A, Ag85B, Ag85C)}

Antigens of the Ag85 complex (Ag85A, Ag85B, Ag85C) have been extensively studied and were included in a number of studies selected in this review. In the newer studies those antigens were mostly included as "classical" and "reference" antigens (29, 39, 41, 48), however three older studies, recruiting patients in South America reported more in-depth results $(18,19,49)$. Schwander et al. reported stimulation with $\mathrm{Ag} 85 \mathrm{~A}$ and $\mathrm{Ag} 85 \mathrm{~B}$ resulted in significantly higher IFN- $\gamma$ producing $\mathrm{T}$ cells in both PBMCs and broncho-alveolar cells of household contacts in a short-term assay compared to healthy controls (49). Antas et al. measured the immune response after stimulation with $\mathrm{Ag} 85 \mathrm{~A}$ and $\mathrm{Ag} 85 \mathrm{~B}$ in the course of anti-mycobacterial treatment in aTB patients and found IFN $-\gamma$ concentrations to be higher in treated patients and community controls compared to untreated patients (19). Alvarez-Corales et al. reported that in addition to IFN- $\gamma$ also IL17 production was increased in exposed non-household contacts compared to aTB patients when stimulated with Ag85A and Ag85B (18).

\section{Other Antigens}

The secreted protein Rv1860 was found to induce higher frequencies of polyfunctional $\mathrm{T}$ cells dominated by a CD8 immune response in LTBI compared to aTB patients in a study in India (48).

Alvarez-Corrales explored the potential of several Pro-ProGlu family members in aTB patients and exposed healthcare workers (18). Rv3347 was a strong inducer of IFN- $\gamma$ production in exposed individuals compared to aTB. On the other hand, IL-17 production was significantly higher in the exposed group compared to both aTB and healthy controls for the two antigens Rv0978c and Rv1917c (18).

$\mathrm{Li}$ et al. found that the inclusion of Rv3615c as addition in the commercial ELISPOT test improved sensitivity in detecting aTB patients. However, specificity was slightly lower compared to the commercial T-SPOT.TB test and most importantly the study lacks the inclusion of a LTBI patient group (40).

Pathakumari et al. investigated the role of the secreted proteins Rv0009 and Rv2204c among LTBI and aTB patients in a longterm assay. In both studies they looked at several cytokines and found that IFN- $\gamma$ and IFN- $\gamma /$ TNF- $\alpha$ responses to be the most accurate in identifying LTBI $(45,46)$.

The secreted antigens Rv0455c, Rv1511, and Rv1626 showed promise in a study measuring changes in immune response toward several antigens over treatment course. Bertholet et al. found that these secreted antigens showed changes in at least two different cytokines (IFN- $\gamma$, TNF- $\alpha$, or IL-10) in aTB and associated this with treatment success (24).

\section{DISCUSSION}

Our review is, to our knowledge, the first to systematically review and summarize published evidence on the use of novel antigens for the diagnosis of TB. Although a large number of studies were screened for this review, 34 studies only were included in the final analysis. The main reason leading to the exclusion of many of the screened studies was the lack of detailed information in the particular studies relating to study population characteristics such as age, gender and immune status of the participants. In addition, clear disease classification for both LTBI and aTB was commonly missing as well as information whether patients have been started on treatment before the novel antigens were tested and what the prior duration of treatment was. Stringent inclusion criteria were therefore used in this review to enable comparison between studies and summarize evidence on novel antigens most promising to improve diagnosis before treatment initiation.

One of the most striking findings is that most of the tested novel antigens belong to the group of latency associated antigens, in particular from the DosR regulon. The DosR regulon is a specific region of the M. tuberculosis genome comprising approximately 50 genes that are activated during the dormant, non-replicative state (53). In general, the observed immune responses induced by these antigens are more pronounced in patients with LTBI compared to aTB, making these antigens attractive for distinction of infection vs. disease (54). Further to their potential for discrimination of LTBI and aTB these antigens may also be useful for monitoring treatment success in aTB patients.

The most promising candidate M. tuberculosis DosR regulon encoded antigens were Rv0081, Rv1733c, Rv1737c, Rv2029c, Rv2628, which showed high immunogenic potential across studies and geographical regions $(20-22,26,27,29,35,38,39$, 42).

Rv0081 is a transcriptional regulator (55) and presumably a key locus within the DosR regulon under hypoxic conditions (56). Several studies using long-term incubation in South Africa 
and Ethiopia showed the immunogenic potential of this antigen $(26,27,39)$. However, Kassa et al. only included one groupbeing aTB patients-and both studies by Chegou et al. did not report tuberculin skin test or interferon gamma-release assays results for the LTBI patient groups. Hozumi et al. showed good immunogenic potential but addition of Rv0081 in a classic ELISPOT assay did not result in IFN- $\gamma$ responses able to differentiate LTBI vs. aTB (38).

Rv1733c is presumed to be a transmembrane protein and found to be a highly potent $\mathrm{T}$ cell antigen using bioinformatic analysis by Zvi et al. (15) and Lew et al. (57). Indeed, the studies in our review show that this antigen elicits a higher immune response in LTBI patients compared to aTB and healthy controls. This is in agreement with serval other studies, which were excluded from the review, that the immune response to Rv1733c is potentially a good marker for LTBI patients $(54,58-$ 60 ). However, only the study of Serra-Vidal et al. was able to show the discriminatory potential of Rv1733c both in short- and long-term stimulation assays (60).

Rv1737c is a possible nitrate transporter (61). Four studies in this review assessed its immunogenicity in long-term stimulation assays showing increased response in LTBI $(21,26,27)$ but not aTB patients (39). An elevated immune response in LTBI patients was also described in further studies excluded from the review $(62,63)$ including in two studies investigating exposed individuals across several African sites $(58,64)$.

Rv2029c is thought to be a phosphofructokinase involved in glycolysis (65) and has been tested in mice as potential vaccine candidate antigen (66). It was one of the most widely used antigens among the studies in our review and the only antigen with concordant results across studies in short- and longterm assays. This is in agreement with several other studies not included in the review $(54,62-64)$.

Rv2628 was amongst the top ranking 45 antigens from a list of 189 antigens by Zvi et al. (15). However, its function is not yet clear (57). Many studies in this review employing a variety of different assays showed increased immune responses in LTBI compared to aTB patients $(20,22,35,42)$. Results from other studies not included in this review further highlight the importance of this antigen $(54,62,63)$.

A further antigen from the DosR regulon is Rv2031c, also called $\alpha$-crystallin or heat shock protein $\mathrm{X}$, which has been described as crucial for growth of M. tuberculosis inside macrophages during latency $(67,68)$. Studies in mice and macrophage models have demonstrated its role during $\mathrm{M}$. tuberculosis infection $(67,69,70)$. The findings of the human studies included in our review are less clear with some studies reporting significantly higher cytokine production in exposed individuals and LTBI compared to aTB patients and controls.

Beyond the DosR regulon HBHA was a further latency associated antigen included in several studies for diagnosis of LTBI. HBHA is a protein that mediates dissemination of TB through its binding to epithelial cells at the site of primary infection, a process believed to be key for developing latency $(71,72)$. Concentrations of cytokines were significantly higher in LTBI compared to aTB patients in a number of studies (25,
$30,31,34,37,41,50,51)$. Further to this a few studies including human immunodeficiency virus-infected individuals showed low or absent response to HBHA which may correlate with the risk for disease progression $(25,31)$. Interestingly in a study not included in this review HBHA-induced IFN- $\gamma$ was detectable at the site of infection of TB disease in the absence of a response in the blood, suggesting that novel antigens might also be useful as stimulating antigens in samples from other body sites. As seen for several other antigens the HBHA-specific cytokine readout likely plays an important role as shown in a study by Molicotti et al. in which the ratio of IFN $-\gamma$ and TNF- $\alpha$ was able to distinguish LTBI from aTB patients (73).

Further to the latency associated antigens a smaller number of studies evaluated Rpfs for in-vitro stimulation. Rpfs are a group of proteins involved in the reactivation of non-growing mycobacteria. The proteins are attached to the M. tuberculosis cell wall or secreted rendering them an optimal target for the immune system (74). The studies included in our review suggest that the immune response toward Rv0867c and Rv2389c are potent discriminators of disease stages. Reactivation is a key topic in $\mathrm{TB}$ research and therefore the number of studies investigating these antigens included in this review is surprisingly low. It is important to note that a few further studies investigating these antigens were not included as they did not fulfill the inclusion criteria. Results from the non-included studies showed similar results with Rv2389c $(60,63,75,76)$ and Rv0867c $(63,64,75,76)$ being identified as potent inducers of IFN- $\gamma$ responses in LTBI compared to aTB patients and healthy controls.

Many studies report results using antigens from the Ag85 complex (Ag85A, Ag85B, Ag85C) with consistently elevated cytokine responses in LTBI compared to aTB. The Ag85 complex consists of three secreted proteins that are associated with virulence and crucial for survival of $M$. tuberculosis in macrophages (77). Due to its immune-dominant ability Ag85 has gained interest in vaccine research in recent years. There are currently vaccine candidates in clinical research mostly combining the existing BCG vaccine and additionally overexpressing a protein of the Ag85 complex (78). If successful as vaccines, the inclusion of one of these proteins in a novel diagnostic test could therefore interfere with the diagnosis as a result of cross-reactivity in a vaccinated population.

Apart from the inclusion of novel antigens, many studies extend the measurement of cytokines beyond IFN- $\gamma$ to improve diagnostic assays $(79,80)$. Approximately half of the studies included in this review measured additional cytokines with IL2 , IL-10, IL-17, and TNF- $\alpha$ being among the most frequently used ones that improved diagnostic potential. A recent study by Coppola et al, not reviewed here, provided evidence that almost half of the new M. tuberculosis antigens tested in LTBI populations did trigger many other cytokines than IFN- $\gamma$, and often no IFN- $\gamma$ itself (16).

One limitation of our review is that despite stringent inclusion criteria, there is inevitable variability between studies particularly for the patients included in the study groups. Whereas, the definitions for aTB patients were mostly consistent, there is considerable heterogeneity in the definition of LTBI patients. This often resulted from local routine practice of standard 
LTBI diagnosis. One further limitation is that some of the antigens have already been tested extensively and information on function and location are available. For others this information is still unknown, which renders interpretation of study results speculative in some instances.

\section{CONCLUSIONS}

In our review compiling the latest research from novel M. tuberculosis antigens several studies clearly showed the discriminatory potential of LTBI from aTB of a number of antigens with the most promising being latency associated antigens. Findings for these antigens are consistent across several studies with immune responses detectable in shortterm incubation periods. Moreover, the inclusion of additional cytokine read-outs complementing IFN- $\gamma$ results, appears to increase discriminatory potential of LTBI from aTB. Despite these considerable advances in recent years especially children and immune-compromised patients are highly underrepresented in these studies and further research investigating novel antigens in these patient groups need to address this issue.

\section{REFERENCES}

1. World Health Organization. Global Tuberculosis Report 2017. Geneva (2017).

2. United Nations. The Sustainable Development Goals Report 2017. New York, NY (2017).

3. Ritz N, Curtis N. Novel concepts in the epidemiology, diagnosis and prevention of childhood tuberculosis. Swiss Med Wkly. (2014) 144:w14000. doi: 10.4414/smw.2014.14000

4. Huebner RE, Schein MF, Bass JB, Jr. The tuberculin skin test. Clin Infect Dis. (1993) 17:968-75. doi: 10.1093/clinids/17.6.968

5. Andersen P, Munk ME, Pollock JM, Doherty TM. Specific immunebased diagnosis of tuberculosis. Lancet (2000) 356:1099-104. doi: 10.1016/S0140-6736(00)02742-2

6. Lalvani A, Pareek M. A 100 year update on diagnosis of tuberculosis infection. Br Med Bull. (2010) 93:69-84. doi: 10.1093/bmb/ldp039

7. Lalvani A, Pathan AA, McShane H, Wilkinson RJ, Latif M, Conlon CP, et al. Rapid detection of Mycobacterium tuberculosis infection by enumeration of antigen-specific T cells. Am J Res Crit Care Med. (2001) 163:824-8. doi: 10.1164/ajrccm.163.4.2009100

8. Harboe M, Oettinger T, Wiker HG, Rosenkrands I, Andersen P. Evidence for occurrence of the ESAT-6 protein in Mycobacterium tuberculosis and virulent Mycobacterium bovis and for its absence in Mycobacterium bovis BCG. Infect Immun. (1996) 64:16-22.

9. Guinn KM, Hickey MJ, Mathur SK, Zakel KL, Grotzke JE, Lewinsohn DM, et al. Individual RD1-region genes are required for export of ESAT-6/CFP10 and for virulence of Mycobacterium tuberculosis. Mol Microbiol. (2004) 51:359-70. doi: 10.1046/j.1365-2958.2003.03844.x

10. World Health Organization W. Latent TB Infection: Updated and Consolidated Guidelines for Programmatic Management (2018).

11. Lu P, Chen X, Zhu LM, Yang HT. Interferon-gamma release assays for the diagnosis of tuberculosis: a systematic review and meta-analysis. Lung (2016) 194:447-58. doi: 10.1007/s00408-016-9872-5

12. Sollai S, Galli L, de Martino M, Chiappini E. Systematic review and meta-analysis on the utility of Interferon-gamma release assays for the diagnosis of Mycobacterium tuberculosis infection in children: a 2013 update. BMC Infect Dis. (2014) 14 (Suppl .1)S6. doi: 10.1186/1471-233414-S1-S6

13. Pai M, Zwerling A, Menzies D. Systematic review: T-cell-based assays for the diagnosis of latent tuberculosis infection: an update. Ann Intern Med. (2008) 149:177-184. doi: 10.7326/0003-4819-149-3-200808050-00241

\section{AUTHOR CONTRIBUTIONS}

NM designed the search strategy. NR reviewed and approved the search strategy. NM searched the literature, selected the studies, extracted, and analyzed the data. NR controlled the quality of the review process. NM drafted the initial manuscript. NR, MJ, and TO reviewed and edited the manuscript. All authors read and approved the final manuscript.

\section{ACKNOWLEDGMENTS}

NM was supported by the following associations: Bangerter Rhyner Stiftung, Lunge Zürich, Nora van Meeuwen-Häftliger Stiftung, Rozalia Foundation and Schweizerische Lungenstiftung.

\section{SUPPLEMENTARY MATERIAL}

The Supplementary Material for this article can be found online at: https://www.frontiersin.org/articles/10.3389/fimmu. 2018.02476/full\#supplementary-material

14. Geluk A, van Meijgaarden KE, Joosten SA, Commandeur S, Ottenhoff TH. Innovative Strategies to Identify M. tuberculosis Antigens and Epitopes Using Genome-Wide Analyses. Front Immunol. (2014) 5:256. doi: 10.3389/fimmu.2014.00256

15. Zvi A, Ariel N, Fulkerson J, Sadoff JC, Shafferman A. Whole genome identification of Mycobacterium tuberculosis vaccine candidates by comprehensive data mining and bioinformatic analyses. BMC Med Genomics. (2008) 1:18. doi: 10.1186/1755-8794-1-18

16. Coppola M, van Meijgaarden KE, Franken KL, Commandeur S, Dolganov G, Kramnik I, et al. New genome-wide algorithm identifies novel invivo expressed mycobacterium tuberculosis antigens inducing human T-cell responses with classical and unconventional cytokine profiles. Sci Rep. (2016) 6:37793. doi: 10.1038/srep37793

17. Cole ST, Brosch R, Parkhill J, Garnier T, Churcher C, Harris D, et al. Deciphering the biology of Mycobacterium tuberculosis from the complete genome sequence. Nature (1998) 393:537-44. doi: 10.1038/31159

18. Alvarez-Corrales N, Ahmed RK, Rodriguez CA, Balaji KN, Rivera R, Sompallae R, et al. Differential cellular recognition pattern to $\mathrm{M}$. tuberculosis targets defined by IFN-gamma and IL-17 production in blood from TB + patients from Honduras as compared to health care workers: TB and immune responses in patients from Honduras. BMC Infect Dis. (2013) 13:125. doi: 10.1186/1471-2334-13-125

19. Antas PR, Cardoso FL, Pereira KC, Franken KL, Cunha KS, Klatser P, et al. $\mathrm{T}$ cell immune responses to mycobacterial antigens in Brazilian tuberculosis patients and controls. Trans R Soc Trop Med Hygiene (2005) 99:699-707. doi: 10.1016/j.trstmh.2005.05.002

20. Araujo LS, da Silva Nde B, da Silva RJ, Leung JA, Mello FC, Saad MH. Profile of interferon-gamma response to latency-associated and novel in vivo expressed antigens in a cohort of subjects recently exposed to Mycobacterium tuberculosis. Tuberculosis (2015) 95:751-7. doi: 10.1016/j.tube.2015. 08.002

21. Arroyo L, Rojas M, Franken K, Ottenhoff THM, Barrera LF. Multifunctional $\mathrm{T}$ Cell response to DosR and Rpf antigens is associated with protection in long-term Mycobacterium tuberculosis-infected individuals in Colombia. Clin Vacc Immunol. (2016) 23:813-24. doi: 10.1128/CVI.00 217-16

22. Bai XJ, Liang Y, Yang YR, Feng JD, Luo ZP, Zhang JX, et al. Potential novel markers to discriminate between active and latent tuberculosis infection in Chinese individuals. Comp Immunol Microbiol Infect Dis. (2016) 44:8-13. doi: 10.1016/j.cimid.2015.11.002 
23. Belay M, Legesse M, Mihret A, Bekele Y, Ottenhoff TH, Franken KL, et al. Pro- and anti-inflammatory cytokines against Rv2031 are elevated during latent tuberculosis: a study in cohorts of tuberculosis patients, household contacts and community controls in an endemic setting. PLoS ONE (2015) 10:e0124134. doi: 10.1371/journal.pone.0124134

24. Bertholet S, Horne DJ, Laughlin EM, Savlov M, Tucakovic I, Coler $\mathrm{RN}$, et al. Effect of chemotherapy on whole-blood cytokine responses to Mycobacterium tuberculosis antigens in a small cohort of patients with pulmonary tuberculosis. Clin Vacc Immunol. (2011) 18:1378-86. doi: 10.1128/CVI.05037-11

25. Chiacchio T, Delogu G, Vanini V, Cuzzi G, De Maio F, Pinnetti C, et al. Immune characterization of the HBHA-specific response in Mycobacterium tuberculosis-infected patients with or without HIV infection. PLoS ONE (2017) 12:e0183846. doi: 10.1371/journal.pone.0183846

26. Chegou NN, Black GF, Loxton AG, Stanley K, Essone PN, Klein MR, et al. Potential of novel Mycobacterium tuberculosis infection phase-dependent antigens in the diagnosis of TB disease in a high burden setting. BMC Infect Dis. (2012) 12:10. doi: 10.1186/1471-2334-12-10

27. Chegou NN, Essone PN, Loxton AG, Stanley K, Black GF, van der Spuy GD, et al. Potential of host markers produced by infection phase-dependent antigen-stimulated cells for the diagnosis of tuberculosis in a highly endemic area. PLoS ONE (2012) 7:e38501. doi: 10.1371/journal.pone.0038501

28. Chen J, Su X, Zhang Y, Wang S, Shao L, Wu J, et al. Novel recombinant $\mathrm{RD} 2-$ and RD11-encoded Mycobacterium tuberculosis antigens are potential candidates for diagnosis of tuberculosis infections in BCG-vaccinated individuals. Microb Infect. (2009) 11:876-85. doi: 10.1016/j.micinf.2009.05.008

29. Commandeur S, Lin MY, van Meijgaarden KE, Friggen AH, Franken KL, Drijfhout JW, et al. Double- and monofunctional CD4(+) and CD8(+) Tcell responses to Mycobacterium tuberculosis DosR antigens and peptides in long-term latently infected individuals. Eur J Immunol. (2011) 41:2925-36. doi: 10.1002/eji.201141602

30. Delogu G, Chiacchio T, Vanini V, Butera O, Cuzzi G, Bua A, et al. Methylated HBHA produced in M. smegmatis discriminates between active and non-active tuberculosis disease among RD1-responders. PLOS ONE (2011) 6:e0018315. doi: 10.1371/journal.pone.0018315

31. Delogu G, Vanini V, Cuzzi G, Chiacchio T, De Maio F, Battah B, et al. Lack of response to HBHA in $\mathrm{HIV}$-infected patients with latent tuberculosis infection. Scandinavian J Immunol. (2016) 84:344-52. doi: 10.1111/sji.12493

32. Doddam SN, Peddireddy V, Ahmed N. Mycobacterium tuberculosis DosR regulon gene rv2004c encodes a novel antigen with pro-inflammatory functions and potential diagnostic application for detection of latent tuberculosis. Front Immunol. (2017) 8:712. doi: 10.3389/fimmu.2017.00712

33. Dosanjh DP, Bakir M, Millington KA, Soysal A, Aslan Y, Efee S, et al. Novel M tuberculosis antigen-specific T-cells are early markers of infection and disease progression. PLoS ONE (2011) 6:e28754. doi: 10.1371/journal.pone.0028754

34. Dreesman A, Corbiere V, Dirix V, Smits K, Debulpaep S, De Schutter I, et al. Age-Stratified T Cell Responses in Children Infected with Mycobacterium tuberculosis. Front Immunol. (2017) 8:e01059. doi: 10.3389/fimmu.2017.01059

35. Goletti D, Butera O, Vanini V, Lauria FN, Lange C, Franken KL, et al. Response to Rv2628 latency antigen associates with cured tuberculosis and remote infection. Eur Res J. (2010) 36:135-42. doi: 10.1183/09031936.00140009

36. Govender L, Abel B, Hughes EJ, Scriba TJ, Kagina BM, de Kock $\mathrm{M}$, et al. Higher human CD4 T cell response to novel Mycobacterium tuberculosis latency associated antigens Rv2660 and Rv2659 in latent infection compared with tuberculosis disease. Vaccine (2010) 29:51-7. doi: 10.1016/j.vaccine.2010.10.022

37. Hougardy JM, Schepers K, Place S, Drowart A, Lechevin V, Verscheure $\mathrm{V}$, et al. Heparin-binding-hemagglutinin-induced IFN- $\gamma$ release as a diagnostic tool for latent tuberculosis. PLoS ONE (2007) 2:e0000926. doi: 10.1371/journal.pone.0000926

38. Hozumi H, Tsujimura K, Yamamura Y, Seto S, Uchijima M, Nagata T, et al. Immunogenicity of dormancy-related antigens in individuals infected with Mycobacterium tuberculosis in Japan. Int J Tuberc Lung Dis. (2013) 17:818-24. doi: 10.5588 /ijtld.12.0695

39. Kassa D, Ran L, Geberemeskel W, Tebeje M, Alemu A, Selase A, et al. Analysis of immune responses against a wide range of Mycobacterium tuberculosis antigens in patients with active pulmonary tuberculosis. Clin Vacc Immunol. (2012) 19:1907-15. doi: 10.1128/CVI.00482-12

40. Li G, Li F, Zhao HM, Wen HL, Li HC, Li CL, et al. Evaluation of a New IFN-gamma release assay for rapid diagnosis of active tuberculosis in a high-incidence setting. Front Cell Infect Microbiol. (2017) 7:117. doi: $10.3389 /$ fcimb.2017.00117

41. Loxton AG, Black GF, Stanley K, Walzl G. Heparin-binding hemagglutinin induces IFN-gamma(+) IL-2(+) IL-17(+) multifunctional CD4(+) T cells during latent but not active tuberculosis disease. Clin Vacc Immunol. (2012) 19:746-51. doi: 10.1128/CVI.00047-12

42. Mensah GI, Addo KK, Tetteh JA, Sowah S, Loescher T, Geldmacher C, et al. Cytokine response to selected MTB antigens in Ghanaian TB patients, before and at 2 weeks of anti-TB therapy is characterized by high expression of IFNgamma and Granzyme B and inter- individual variation. BMC Infect Dis. (2014) 14:495. doi: 10.1186/1471-2334-14-495

43. Michelsen SW, Soborg B, Agger EM, Diaz LJ, Hoff ST, Koch A, et al. Host immunity to Mycobacterium tuberculosis and risk of tuberculosis: A longitudinal study among Greenlanders. Vaccine (2016) 34:5975-83. doi: 10.1016/j.vaccine.2016.09.047

44. Michelsen SW, Soborg B, Diaz LJ, Hoff ST, Agger EM, Koch $\mathrm{A}$, et al. The dynamics of immune responses to Mycobacterium tuberculosis during different stages of natural infection: A longitudinal study among Greenlanders. Plos ONE (2017) 12:e0177906. doi: 10.1371/journal.pone.0177906

45. Pathakumari B, Anbarasu D, Parthasarathy RT, Raja A. PpiA antigen specific immune response is a potential biomarker for latent tuberculosis infection. Tuberculosis (2015) 95:736-43. doi: 10.1016/j.tube.2015.07.006

46. Pathakumari B, Prabhavathi M, Raja A. Evaluation of cytokine and chemokine response elicited by Rv2204c and Rv0753c to detect latent tuberculosis infection. Cytokine (2015) 76:496-504. doi: 10.1016/j.cyto.2015. 07.028

47. Peña D, Rovetta AI, Hernandez Del Pino RE, Amiano NO, Pasquinelli V, Pellegrini JM, et al. A Mycobacterium tuberculosis dormancy antigen differentiates latently infected bacillus calmette-guerin-vaccinated individuals. EBioMedicine (2015) 2:884-90. doi: 10.1016/j.ebiom.2015.05.026

48. Satchidanandam V, Kumar N, Biswas S, Jumani RS, Jain C, Rani R, et al The secreted protein Rv1860 of Mycobacterium tuberculosis stimulates human polyfunctional CD8(+) T Cells. Clin Vacc Immunol. (2016) 23:282-93. doi: 10.1128/CVI.00554-15

49. Schwander SK, Torres M, Carranza CC, Escobedo D, Tary-Lehmann M, Anderson P, et al. Pulmonary mononuclear cell responses to antigens of Mycobacterium tuberculosis in healthy household contacts of patients with active tuberculosis and healthy controls from the community. J Immunol. (2000) 165:1479-85. doi: 10.4049/jimmunol.165.3.1479

50. Wyndham-Thomas C, Corbiere V, Dirix V, Smits K, Domont F, Libin $\mathrm{M}$, et al. Key role of effector memory CD4+ $\mathrm{T}$ lymphocytes in a shortincubation heparin-binding hemagglutinin gamma interferon release assay for the detection of latent tuberculosis. Clin Vacc Immunol. (2014) 21:321-8. doi: 10.1128/CVI.00651-13

51. Wyndham-Thomas C, Dirix V, Schepers K, Martin C, Hildebrand M, Goffard JC, et al. Contribution of a heparin-binding haemagglutinin interferongamma release assay to the detection of Mycobacterium tuberculosis infection in HIV-infected patients: Comparison with the tuberculin skin test and the QuantiFERON ${ }^{\circledR}$-TB Gold In-tube. BMC Infect Dis. (2015) 15:59. doi: 10.1186/s12879-015-0796-0

52. Betts JC, Lukey PT, Robb LC, McAdam RA, Duncan K. Evaluation of a nutrient starvation model of Mycobacterium tuberculosis persistence by gene and protein expression profiling. Mol Microbiol. (2002) 43:717-31. doi: 10.1046/j.1365-2958.2002.02779.x

53. Voskuil MI, Schnappinger D, Visconti KC, Harrell MI, Dolganov GM, Sherman DR, et al. Inhibition of respiration by nitric oxide induces a Mycobacterium tuberculosis dormancy program. J Exp Med. (2003) 198:70513. doi: 10.1084/jem.20030205

54. Leyten EM, Lin MY, Franken KL, Friggen AH, Prins C, van Meijgaarden $\mathrm{KE}$, et al. Human T-cell responses to 25 novel antigens encoded by genes of the dormancy regulon of Mycobacterium tuberculosis. Microb Infect. (2006) 8:2052-60. doi: 10.1016/j.micinf.2006.03.018 
55. He H, Bretl DJ, Penoske RM, Anderson DM, Zahrt TC. Components of the Rv0081-Rv0088 locus, which encodes a predicted formate hydrogenlyase complex, are coregulated by Rv0081, MprA, and DosR in Mycobacterium tuberculosis. J Bacteriol. (2011) 193:5105-18. doi: 10.1128/JB. 05562-11

56. Galagan JE, Minch K, Peterson M, Lyubetskaya A, Azizi E, Sweet L, et al. The Mycobacterium tuberculosis regulatory network and hypoxia. Nature (2013) 499:178-83. doi: 10.1038/nature12337

57. Lew JM, Kapopoulou A, Jones LM, Cole ST. TubercuList-10 years after. Tuberculosis (2011) 91:1-7. doi: 10.1016/j.tube.2010.09.008

58. Black GF, Thiel BA, Ota MO, Parida SK, Adegbola R, Boom WH, et al. Immunogenicity of novel DosR regulon-encoded candidate antigens of Mycobacterium tuberculosis in three high-burden populations in Africa. Clin Vacc Immunol. (2009) 16:1203-12. doi: 10.1128/CVI.00111-09

59. Schuck SD, Mueller H, Kunitz F, Neher A, Hoffmann H, Franken K, et al. Identification of T-cell antigens specific for latent Mycobacterium Tuberculosis infection. Plos ONE (2009) 4:e0005590. doi: 10.1371/journal.pone.0005590

60. Serra-Vidal MM, Latorre I, Franken KL, Diaz J, de Souza-Galvao ML, Casas I, et al. Immunogenicity of 60 novel latency-related antigens of Mycobacterium tuberculosis. Front Microbiol. (2014) 5:517. doi: 10.3389/fmicb.2014.00517

61. Hutter B, Dick T. Analysis of the dormancy-inducible narK2 promoter in Mycobacterium bovis BCG. FEMS Microbiol Lett. (2000) 188:141-6. doi: 10.1111/j.1574-6968.2000.tb09185.x

62. Rakshit S, Adiga V, Nayak S, Sahoo PN, Sharma PK, van Meijgaarden KE, et al. Circulating Mycobacterium tuberculosis DosR latency antigen-specific, polyfunctional, regulatory IL10(+) Th17 CD4 T-cells differentiate latent from active tuberculosis. Sci Rep. (2017) 7:11948. doi: 10.1038/s41598-017-10773-5

63. Riano F, Arroyo L, Paris S, Rojas M, Friggen AH, van Meijgaarden $\mathrm{KE}$, et al. $\mathrm{T}$ cell responses to DosR and Rpf proteins in actively and latently infected individuals from Colombia. Tuberculosis (2012) 92:148-59. doi: 10.1016/j.tube.2011.12.005

64. Sutherland JS, Lalor MK, Black GF, Ambrose LR, Loxton AG, Chegou $\mathrm{NN}$, et al. Analysis of host responses to Mycobacterium tuberculosis antigens in a multi-site study of subjects with different TB and HIV infection states in sub-Saharan Africa. PLoS ONE (2013) 8:e74080. doi: 10.1371/journal.pone.0074080

65. Phong WY, Lin W, Rao SP, Dick T, Alonso S, Pethe K. Characterization of phosphofructokinase activity in Mycobacterium tuberculosis reveals that a functional glycolytic carbon flow is necessary to limit the accumulation of toxic metabolic intermediates under hypoxia. PLoS ONE (2013) 8:e56037. doi: 10.1371/journal.pone.0056037

66. Su H, Zhu S, Kong C, Huang Q, Zhang Z, Wang H, et al. Mycobacterium tuberculosis latent antigen Rv2029c from the multistage DNA vaccine A39 drives TH1 responses via TLR-mediated macrophage activation. Front Microbiol. (2017) 8:2266. doi: 10.3389/fmicb.2017.02266

67. Yuan Y, Crane DD, Simpson RM, Zhu YQ, Hickey MJ, Sherman DR, et al. The 16-kDa alpha-crystallin (Acr) protein of Mycobacterium tuberculosis is required for growth in macrophages. Proc Natl Acad Sci USA. (1998) 95:9578-83. doi: 10.1073/pnas.95.16.9578

68. Yuan Y, Crane DD, Barry CE, 3rd. Stationary phase-associated protein expression in Mycobacterium tuberculosis: function of the mycobacterial alpha-crystallin homolog. J Bacteriol. (1996) 178:4484-92. doi: 10.1128/jb.178.15.4484-4492.1996

69. Hu Y, Movahedzadeh F, Stoker NG, Coates AR. Deletion of the Mycobacterium tuberculosis alpha-crystallin-like hspX gene causes increased bacterial growth in vivo. Infect Immun. (2006) 74:861-8. doi: 10.1128/IAI.74.2.861-868.2006

70. Hu Y, Coates AR. Transcription of the stationary-phase-associated hspX gene of Mycobacterium tuberculosis is inversely related to synthesis of the 16-kilodalton protein. J Bacteriol. (1999) 181:1380-7.

71. Pethe K, Alonso S, Biet F, Delogu G, Brennan MJ, Locht C, et al. The heparinbinding haemagglutinin of $\mathrm{M}$. tuberculosis is required for extrapulmonary dissemination. Nature (2001) 412:190-4. doi: 10.1038/35084083

72. Locht C, Hougardy JM, Rouanet C, Place S, Mascart F. Heparin-binding hemagglutinin, from an extrapulmonary dissemination factor to a powerful diagnostic and protective antigen against tuberculosis. Tuberculosis (2006) 86:303-9. doi: 10.1016/j.tube.2006.01.016

73. Molicotti P, Bua A, Cubeddu M, Cannas S, Delogu G, Zanetti S. Tuberculosis patients are characterized by a low-IFN-gamma/high-TNF-alpha response to methylated HBHA produced in M. smegmatis. Diagnost Microbiol Infect Dis. (2011) 71:449-52. doi: 10.1016/j.diagmicrobio.2011.08.010

74. Rosser A, Stover C, Pareek M, Mukamolova GV. Resuscitationpromoting factors are important determinants of the pathophysiology in Mycobacterium tuberculosis infection. Crit Rev Microbiol. (2017) 43:621-30. doi: 10.1080/1040841X.2017.1283485

75. Huang W, Qi Y, Ren C, Wen H, Franken KL, Ottenhoff TH, et al. Interferon-gamma responses to Mycobacterium tuberculosis Rpf proteins in contact investigation. Tuberculosis (2013) 93:612-7. doi: 10.1016/j.tube.2013. 08.005

76. Commandeur S, van Meijgaarden KE, Lin MY, Franken K, Friggen $\mathrm{AH}$, Drijfhout JW, et al. Identification of Human T-Cell Responses to Mycobacterium tuberculosis resuscitation-promoting factors in longterm latently infected individuals. Clin Vacc Immunol. (2011) 18:676-83. doi: 10.1128/CVI.00492-10

77. Babaki MKZ, Soleimanpour S, Rezaee SA. Antigen 85 complex as a powerful Mycobacterium tuberculosis immunogene: biology, immune-pathogenicity, applications in diagnosis, and vaccine design. Microb Pathog. (2017) 112:20-9. doi: 10.1016/j.micpath.2017.08.040

78. Hatherill M, Tait D, McShane H. Clinical testing of tuberculosis vaccine candidates. Microbiol Spect. (2016) 4:1-18 doi: 10.1128/microbiolspec.TBTB2-0015-2016

79. Walzl G, Ronacher K, Hanekom W, Scriba TJ, Zumla A. Immunological biomarkers of tuberculosis. Nat Rev Immunol. (2011) 11:343-54. doi: $10.1038 /$ nri2960

80. Ferrara G, Losi M, Fabbri LM, Migliori GB, Richeldi L, Casali L. Exploring the immune response against Mycobacterium tuberculosis for a better diagnosis of the infection. Arch Immunol et Therapiae Expe. (2009) 57:425-33. doi: 10.1007/s00005-009-0050-9

Conflict of Interest Statement: The authors declare that the research was conducted in the absence of any commercial or financial relationships that could be construed as a potential conflict of interest.

Copyright (C) 2018 Meier, Jacobsen, Ottenhoff and Ritz. This is an open-access article distributed under the terms of the Creative Commons Attribution License (CC BY). The use, distribution or reproduction in other forums is permitted, provided the original author(s) and the copyright owner(s) are credited and that the original publication in this journal is cited, in accordance with accepted academic practice. No use, distribution or reproduction is permitted which does not comply with these terms. 